\title{
Intuitionistic Unbalanced Linguistic Generalized Multiple Attribute Group Decision Making and Its Application to Green Products Selection
}

\author{
Bing Han, ${ }^{1}$ Zhifu Tao, ${ }^{2}$ Huayou Chen $\left(D,{ }^{1}\right.$ and Ligang Zhou ${ }^{1,3}$ \\ ${ }^{1}$ School of Mathematical Sciences, Anhui University, Hefei, Anhui, 230601, China \\ ${ }^{2}$ School of Economics, Anhui University, Hefei, Anhui, 230601, China \\ ${ }^{3}$ China Institute of Manufacturing Development, Nanjing University of Information Science and Technology, Nanjing 210044, China
}

Correspondence should be addressed to Huayou Chen; huayouc@126.com

Received 11 April 2018; Revised 18 July 2018; Accepted 25 July 2018; Published 3 October 2018

Academic Editor: Eric Lefevre

Copyright (C) 2018 Bing Han et al. This is an open access article distributed under the Creative Commons Attribution License, which permits unrestricted use, distribution, and reproduction in any medium, provided the original work is properly cited.

In many countries, green products play a critical role in energy recycling and environment protection. The selection of green products can be regarded as a multiple attribute decision making (MADM) problem. Due to the complexity and uncertainty of the problem, decision makers may give their personal preference values to different attributes of alternatives by intuitionistic unbalanced linguistic term sets. The main purpose of this paper is to put forward a new generalized multiple attribute group decision making (GMAGDM) approach based on the intuitionistic unbalanced linguistic dependent weighted generalized Heronian mean (IULDWGHM) operator and the intuitionistic unbalanced linguistic dependent weighted generalized geometric Heronian mean (IULDWGGHM) operator. The proposed method can not only relieve the influence of unfair assessments, but also consider the interaction effects of attributes. Furthermore, the appropriate parameter values and operators can be selected to meet the different risk preference of decision makers and actual requirements. Finally, a green products selection case is given to illustrate the effectiveness and universality of the developed approach.

\section{Introduction}

Zadeh [1-3] introduced the concept of linguistic variable in 1975. It can deal with qualitative situation in form of words and sentences. For example, the performance of a car is a linguistic term rather than numeric, i.e., very good, good, medium, bad, very bad, quite bad,... and so on. Generally, the linguistic variable is the element of the linguistic term set with uncertain granularity.

Soon afterwords, series linguistic models have been presented to manage decision making problems in uncertain circumstance. Herrera and Martínez [4] initiated the 2-tuple fuzzy linguistic representation model with a linguistic term and a numeric value assessed in $[-0.5,0.5)$, so that the loss of information in the fusion process is avoided. $\mathrm{Xu}$ [5] proposed the virtual linguistic model and defined some operational laws. It overcomes the problem that operational results of linguistic variables exceed bounds of the original linguistic term set. Wang and $\mathrm{Li}[6]$ put forward the concept of the intuitionistic linguistic sets which combines the intuitionistic fuzzy set and the the linguistic set to express the fuzzy information. Rodríguez et.al. [7] introduced the hesitant fuzzy linguistic model to handle conditions where experts may hesitate among several consecutive qualitative linguistic terms. Ji, Zhang and Wang [8] considered the outranking method with multi-heditant fuzzy linguistic term set by introducing the projection. Wang and Peng [9] put forword hesitant linguistic intuitionistic fuzzy sets (HLIFSs) based on hesitant fuzzy sets (HFSs) and linguistic intuitionistic fuzzy numbers (LIFNs) which can depict complex and uncertain decision-making information and reflect the hesitancy of decision-makers. A computational model based on type-2 fuzzy sets was proposed by Mendel and Türkşen [10] which maintained the uncertainty and reduced the computational efforts when aggregating them. Recently, Herrera, et al. [11] presented a new fuzzy linguistic methodology called unbalanced linguistic term sets, in which linguistic labels are non-uniformly and asymmetrically distributed. 
After the concept of unbalanced linguistic term sets was presented, numerous studies have been developed on both theoretical basis and practical applications. Bartczuk, et al. [12] introduced a new methodology to handle unbalanced linguistic information with a linguistic label and a value of the correction factor. Dong, Li and Herrera [13] proposed a novel numerical scale model to address the hesitant fuzzy unbalanced linguistic term sets. Dong, et al. [14] put forward the unbalanced linguistic assessments with interval symbolic proportions under multi-granular linguistic contexts. Wang, Liang and Qian [15] built a normalized numerical scaling approach to determine semantics of multigranular linguistic terms, which can lower the complexity of computation and the subjectivity in transformation process. Jiang, Liu et al. [16] gave an aggregation method for unbalanced fuzzy linguistic information by using the linguistic proportional 2-tuple power average operator, while Mata, et al. [17] proposed the type-1 OWA operator to aggregate linguistic values of unbalanced linguistic terms. Dong, et al $[18,19]$ made studies on the preference relations with unbalanced linguistic information to obtain a required consistency level. By using the 2-tuple model, Wang et al.[20] designed a new onling recommendation model based on unbalanced variables and integrated cloud. More related works can be seen in Refs. [2127]

It is noteworthy that the unbalanced linguistic term sets can represent the saltation and nonlinear performance of human thought. Thus, it has real meaning to study the applications of unbalanced linguistic information. Among these applications, the aggregation of this linguistic variables is of great important. A lot of aggregatopn operators have been introduced in [28-34], Herrera, et al. [11] put forword the arithmetic mean of linguistic 2-tuples to for unbalanced linguistic variables. Isern, et al. [35] utilized the concepts of ordered weighted averaging operators to aggregate unbalanced linguistic variables. Han et al. [36] gave an aggregation method for unbalanced linguistic information by untilizing the generalized Heronian mean operators. Han et al. [37] processed the unbalanced linguistic information with a generalized dependent OWA operator which can relieve the influence of unfair linguistic variables by assigning low weights to the biased ones, and make the decision results more reasonable.

Noting that discussions on MADM problems are always in the situation that the attribute sets faced by decision makers are the same. However, the decision makers may consider attribute sets that are not the same due to the different knowledge background. That is to say, they may make mistakes when they give preference values out of their expertised fields. For example, the government tried to invest a new green product, four suppliers are selected for further consideration. The assessments are provided by four departments. The environment department may consider the recyclability level $\left(a_{1}\right)$, the contamination degree $\left(a_{2}\right)$; the comprehensive department could focus on the public satisfaction $\left(a_{3}\right)$ and company scale $\left(a_{4}\right)$; the technology sector may care about the speed of reusability $\left(a_{5}\right)$, the quality of maintenance $\left(a_{6}\right)$ and the level of technical advice $\left(a_{7}\right)$; the financial department have to think about reasonableness of the charge $\left(a_{8}\right)$. Herein, four departments take into account alternatives via their own attribute information, respectively. Thus it is necessary to consider the generalized MADM (GMADM) in which the decision making attributes are changeable for different decision makers.

Comparing to real number and the fuzzy set, intuitionistic unbalanced linguistic numbers (IULNs), which act as elements of intuitionistic unbalanced linguistic term set, could describe the uncertain and incomplete assessments more effectively. For instance, when the recycling characteristics of a green product is evaluated, the decision maker may state that the product is "Quite Good" with probability of truth, falsity, uncertainty of $40 \%, 30 \%, 30 \%$. The assessment can be expressed as $[Q G,(0.4,0.3)]$ using the IULN. Thus, decision making information is vital to be described by IULNs.

Up to now, the applications of unbalanced linguistic variables in intuitionistic fuzzy situation has not been studied. Besides, the decision makers may assign the high preference values to their preferred alternatives as well as the low evaluation values to their disgusting one. In the meantime, the affecting factors of the green products selection have some relevance, such as the recycling degree and the environment pollution. Based on above analysis, it is very important and necessary to extend the dependent operator and the HM operator to cope with the generalized MAGDM in intuitionistic unbalanced linguistic environment. Thus, the aim of this paper is to solve geen product selection GMAGDM problems in which the evaluation values are correlative intuitionistic unbalanced linguistic information. We will introduce the intuitionistic unbalanced linguistic dependent weighted generalized Heronian mean (IULDWGHM) operator and the intuitionistic unbalanced linguistic dependent weighted generalized geometric Heronian mean(IULDWGGHM) operator by combining the dependent operator and the Heronian mean operator under intuitionistic unbalanced linguistic situations. The most crucial advantages of these operators are that they could take into account correlation of input variables, relieve the influence of unfair assessment values and deal with intuitionistic unbalanced linguistic information. For the situation in which the attribute sets considered by DMs are not identical on account of their different knowledge background, the generalized MAGDM with intuitionistic unbalanced linguistic information is proposed. The constributions of this paper are as follows:

(i) The selection of green products is a generalized multiple attribute group decision making (GMAGDM) problem with intuitionistic unbalanced linguistic numbers due to that the attribute sets provided by decision makers are not identical.

(ii) The unbalanced linguistic representation model and the concept of distance between any two intuitionistic unbalanced linguistic numbers are very convenient to translate the qualitative assessments to quantitative ones.

(iii) The intuitionistic unbalanced linguistic dependent weighted generalized Heronian mean (IULDWGHM) operator and the intuitionistic unbalanced linguistic dependent weighted generalized geometric Heronian mean (IULDWGGHM) operator are proposed to deal with the case of green products selection. The above operators can not only 
relieve the influence of unfair evaluations, but also reflect the relationship of both the different criteria values and the criteria value itself. In addition, it has flexible parameter values, we could select the appropriate parameter values to meet the different actual requirements.

The rest of the paper is arranged as follows: Section 2 introduces some basic concepts and notions. Section 3 proposes a GMAGMD approach for selecting the optimal green product based on intuitionistic unbalanced linguistic dependent weighted generalized Heronian mean operartor and intuitionistic unbalanced linguistic dependent weighted generalized geometric Heronian mean operartor, investigates the properities and some particular cases. Section 4 describes the GMAGDM problem with intuitionistic unbalanced linguistic information, a detailed procedure is proposed for managing the GMAGDM in the following. Subsequently, an example of a green product selecton is given to illustrate the effectiveness and universality of the developed approach in Section 5. Section 6 concludes the comparison analyses with other methods. Finally, the paper is summarized in Section 7.

\section{Preliminaries}

In this section, we briefly review the concepts of the intuitionistic linguistic term set, the unbalanced linguistic term set, the dependent ordered weighted average (DOWA) operator and the Heronian mean (HM) operator.

\subsection{The Intuitionistic Linguistic Set}

Definition 1 (see [6]). Let $X=\left\{x_{1}, \cdots, x_{n}\right\}$ be the universe of discourse. An intuitionistic linguistic term set A on X can be defined as

$$
A=\left\{x_{i}\left[s_{\alpha\left(x_{i}\right),}\left(u_{A}\left(x_{i}\right), v_{A}\left(x_{i}\right)\right)\right] \mid x_{i} \in X\right\}
$$

where $s_{\alpha\left(x_{i}\right)}$ belongs to the continuous linguistic set $\bar{S}$, the function $u_{A}\left(x_{i}\right)$ and $v_{A}\left(x_{i}\right)$ stand for the membership degree and non-membership degree of $x_{i}$ to $s_{\alpha\left(x_{i}\right)}$.

For the sake of convenience, Wang et.al. [6] named $A_{i}=\left[s_{\alpha\left(x_{i}\right)}\left(u_{A}\left(x_{i}\right), v_{A}\left(x_{i}\right)\right)\right]$ an intuitionistic linguistic number (ILN). Some operational laws were given as follows:

Let $A_{1}, A_{2}$ be any two ILNs, $\lambda$ be any positive scalar, then

(i) $A_{1} \oplus A_{2}=\left[s_{\alpha\left(x_{1}\right)+\alpha\left(x_{2}\right)}\right.$,

$$
\left.\left(u\left(x_{1}\right)+u\left(x_{2}\right)-u\left(x_{1}\right) u\left(x_{2}\right), v\left(x_{1}\right) v\left(x_{2}\right)\right)\right]
$$

(ii) $A_{1} \otimes A_{2}=\left[s_{\alpha\left(x_{1}\right) \times \alpha\left(x_{2}\right)}\right.$,

$$
\left.\left(u\left(x_{1}\right) u\left(x_{2}\right), 1-\left(1-v\left(x_{1}\right)\right)\left(1-v\left(x_{2}\right)\right)\right)\right] ;
$$

(iii) $\lambda A_{1}=\left[s_{\lambda \alpha\left(x_{1}\right)},\left(1-\left(1-u\left(x_{1}\right)\right)^{\lambda}, v\left(x_{1}\right)^{\lambda}\right)\right]$

(iv) ${A_{1}}^{\lambda}=\left[s_{\alpha\left(x_{1}\right)^{\lambda}},\left(u\left(x_{1}\right)^{\lambda}, 1-\left(1-v\left(x_{1}\right)\right)^{\lambda}\right)\right]$

Obviously, the results of above operations are still ILNs.
Liu [38] proposed the concept of the score function and the accuracy function of ILNs. Furthermore, the method to compare any two ILNs is proposed as follows.

Definition 2 (see [38]). Let $A_{1}=\left[s_{\alpha\left(x_{1}\right)}\left(u_{A}\left(x_{1}\right), v_{A}\left(x_{1}\right)\right)\right]$ be an ILN, then the score function and the accuracy function of $A_{1}$ can be given as

$$
\begin{aligned}
& S\left(A_{1}\right)=\frac{\alpha\left(x_{1}\right)}{l-1} \times\left(u\left(x_{1}\right)+\frac{1}{2}\left(1-u\left(x_{1}\right)-v\left(x_{1}\right)\right)\right) \\
& H\left(A_{1}\right)=\frac{\alpha\left(x_{1}\right)}{l-1} \times\left(u\left(x_{1}\right)+v\left(x_{1}\right)\right)
\end{aligned}
$$

where $l$ is the granularity of the linguistic term set.

Definition 3 (see [38]). Suppose that $A_{1}=\left[s_{\alpha\left(x_{1}\right)}\left(u_{A}\left(x_{1}\right)\right.\right.$, $\left.\left.v_{A}\left(x_{1}\right)\right)\right] A_{2}=\left[s_{\alpha\left(x_{2}\right)}\left(u_{A}\left(x_{2}\right), v_{A}\left(x_{2}\right)\right)\right]$ are any two ILNs, then

(1) If $S\left(A_{1}\right)>S\left(A_{2}\right)$, then $A_{1}>A_{2}$;

(2) If $S\left(A_{1}\right)=S\left(A_{2}\right)$ and $H\left(A_{1}\right)>H\left(A_{2}\right)$, then $A_{1}>A_{2}$.

Definition 4 (see [38]). Assume that $A_{1}=\left[s_{\alpha\left(x_{1}\right)}\left(u_{A}\left(x_{1}\right)\right.\right.$, $\left.\left.v_{A}\left(x_{1}\right)\right)\right], A_{2}=\left[s_{\alpha\left(x_{2}\right)}\left(u_{A}\left(x_{2}\right), v_{A}\left(x_{2}\right)\right)\right]$ are any two ILNs, then the Hamming distance between $A_{1}$ and $A_{2}$ is defined as

$$
\begin{aligned}
& d\left(A_{1}, A_{2}\right)=\frac{1}{2(l-1)} \times \mid\left(1+u\left(x_{1}\right)-v\left(x_{1}\right)\right) \alpha\left(x_{1}\right) \\
& -\left(1+u\left(x_{2}\right)-v\left(x_{2}\right)\right) \alpha\left(x_{2}\right) \mid
\end{aligned}
$$

2.2. The Unbalanced Linguistic Representation Model. Herrera et.al [11] introduced the concept of the unbalanced linguistic term set to reflect the jumpy of human thinking, i.e., the linguistic assessment variables are non-uniformly and non-symmetrically distributed.

Definition 5 (see [11]). An unbalanced linguistic term set S can be expressed as

$$
S=S_{L} \cup S_{C} \cup S_{R}
$$

where $S_{L}$ is the set of all left labels of the central label, $S_{C}$ contains the central label merely, $S_{R}$ is the set of all right labels of the central one.

Example 6. An unbalanced linguistic term set with 9 granularity $S=\{\mathrm{N}($ none), $\mathrm{NG}$ (not good), $\mathrm{M}$ (middle), AG(almost good), G(good), QG(quite good), VG(very good), AT(almost total), $\mathrm{T}$ (total)\}, which is used to evaluate the comprehensive quality of cars. We obtain $S_{L}=\{N, N G\}, S_{C}=\{M\}, S_{R}=$ $\{A G, G, Q G, V G, A T, T\}$. It can be seen that there are fewer labels in $S_{L}$. The distribution of the semantics can be shown in Figure 1.

The model to handle unbalanced linguistic information is based on linguistic hierarchies and the 2-tuple model. The semantic representation of unbalanced linguistic terms are derived via linguistic hierarchies and the computational model based on 2-tuple is defined to accomplish process of computing with words. 


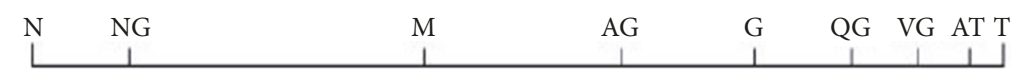

FIGURE 1: Grading system assessments with S.

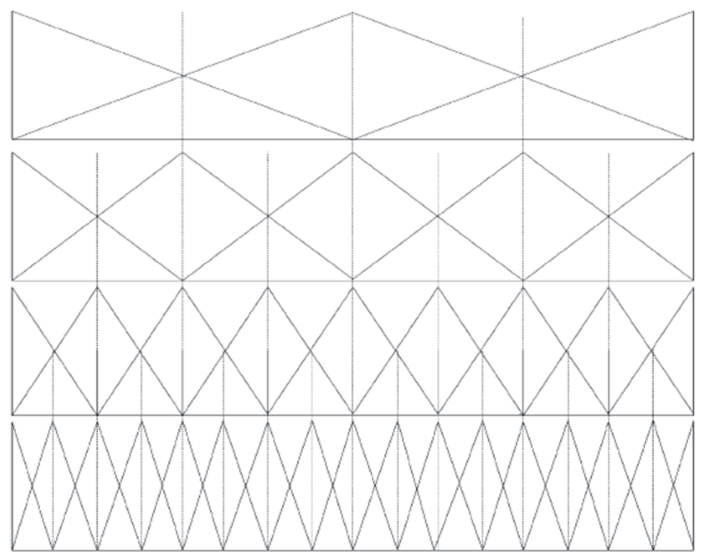

FIgURE 2: Linguistic hierarches of 3, 5, 9 and 17 labels.

TABLE 1: Linguistic hierarches.

\begin{tabular}{lc}
\hline level & granularity \\
\hline $\mathrm{t}=1$ & $\mathrm{n}(\mathrm{t})=3$ \\
$\mathrm{t}=2$ & $\mathrm{n}(\mathrm{t})=5$ \\
$\mathrm{t}=3$ & $\mathrm{n}(\mathrm{t})=9$ \\
$\mathrm{t}=4$ & $\mathrm{n}(\mathrm{t})=17$ \\
\hline
\end{tabular}

A linguistic hierarchy $[39,40]$ is a set of levels where each level is a linguistic term set with a different granularity from the remaining levels of the hierarchy. Each level belonging to a linguistic hierarchy is denoted as $l(t, n(t))$ with $t$ being a number that indicates the level of the hierarchy and $n(t)$ the granularity of the linguistic term set of the $t$ level. For example, a linguistic hierarchy of level 4 is represented by $l(1,3) \cup l(2,5) \cup l(3,9) \cup l(4,17)=\left\{s_{0}^{3}, s_{1}^{3}, s_{2}^{3}\right\} \cup\left\{s_{0}^{5}, \cdots, s_{4}^{5}\right\} \cup$ $\left\{s_{0}^{9}, \cdots, s_{8}^{9}\right\} \cup\left\{s_{0}^{17}, \cdots, s_{16}^{17}\right\}$, its graphics is shown in Figure 2 and Table 1.

In a linguistic hierarchy, the transformation functions (TF) [41] between variables from different levels is defined as follows.

$$
\begin{aligned}
& T F_{t^{\prime}}^{t}\left(s_{i}^{n(t)}, \alpha^{n(t)}\right) \\
& \quad=\Delta_{t^{\prime}}\left(\frac{\Delta_{t}^{-1}\left(s_{i}^{n(t)}, \alpha^{n(t)}\right) \cdot\left(n\left(t^{\prime}\right)-1\right)}{n(t)-1}\right)
\end{aligned}
$$

For example: $T F_{2}^{3}\left(s_{7}^{9}, 0.6\right)=\Delta_{2}\left(\Delta_{3}^{-1}\left(s_{7}^{9}, 0.6\right) \cdot(5-1) /(9-1)\right)=$ $\Delta_{2}(3.8)=\left(s_{4}^{5},-0.2\right)$.

Any unbalanced linguistic 2-tuple $\left(s_{i}, \alpha_{i}\right)$ can be transformed into the term in linguistic hierarchies by the following unbalanced linguistic transformation function $\mathrm{LH}$ and vice versa. The detailed transformation process is given as follows.
(1) Representation of unbalanced linguistic terms in linguistic hierarchies. To accomplish the process of computing with words, the first step is to transform the unbalanced linguistic information in $S$ into the term in linguistic hierarchies. The 2-tuple in linguistic hierarchies associating with respective unbalanced linguistic 2-tuple can be obtained by unbalanced linguistic transformation function $\mathrm{LH}$, i.e.

$$
L H: S \times[-0.5,0.5) \longrightarrow L H \times[-0.5,0.5),
$$

such that $L H\left(s_{i}, \alpha_{i}\right)=\left(s_{k}^{n(t)}, \alpha_{i}\right)$.

(2) Computational process. The process of computing with words can be accomplished by the computation model in linguistic hierarchies. To obtain the expression in the same linguistic domains, we first translate $\left(s_{k}^{n(t)}, \alpha_{i}\right)$ into linguistic 2-tuple in the maximum level $t_{H}$ of linguistic hierarchies, noted as $\left(s_{k^{\prime}}^{n(t)}, \alpha^{\prime}\right)$ by the transformation function TF Eq.(10). Then the linguistic 2-tuple computation model is used with the result as $\left(s_{r}^{n\left(t_{H}\right)}, \alpha_{r}\right)$.

(3) Retransformation phase in unbalanced linguistic term set. By the retransformation phase, the result $\left(s_{r}^{n\left(t_{H}\right)}, \alpha_{r}\right)$ is translated into the unbalanced linguistic term via the transformation function $\mathrm{LH}^{-1}$ :

$$
L H^{-1}: L H \times[-0.5,0.5) \longrightarrow S \times[-0.5,0.5),
$$

such that $L H^{-1}\left(s_{r}^{n\left(t_{H}\right)}, \alpha_{r}\right)=\left(s_{\text {result }}, \lambda_{\text {result }}\right), \lambda_{\text {result }}$ can be determined by cases as follows:

Case 1. If $s_{i}\left(s_{i} \in S\right)$ is represented by merely one label in $\mathrm{LH}$, then $L H^{-1}\left(s_{r}^{n\left(t_{H}\right)}, \alpha_{r}\right)=\left(s_{i}, \alpha_{r}\right)$, that is $\lambda_{\text {result }}=\alpha_{r}$; 
Case 2. If $s_{i}\left(s_{i} \in S\right)$ is represented by two labels in LH, then $\lambda_{\text {result }}=\alpha_{r}$ or

$$
\begin{aligned}
& \lambda_{\text {result }} \\
& \qquad \begin{aligned}
= & \frac{\Delta_{t}^{-1}\left(s_{r}^{n\left(t_{H}\right)}, \alpha_{r}\right) \cdot\left(n\left(t_{H}+1\right)-1\right)}{n(t)-1} \\
& -\operatorname{round}\left(\frac{\Delta_{t}^{-1}\left(s_{r}^{n\left(t_{H}\right)}, \alpha_{r}\right) \cdot\left(n\left(t_{H}+1\right)-1\right)}{n(t)-1}\right)
\end{aligned}
\end{aligned}
$$

Case 3. If there exists no $s_{i} \in S$ such that $s_{i}=s_{r}^{n\left(t_{H}\right)}$, we convert $s_{r}^{n\left(t_{H}\right)}$ into another level, that is

$L H^{-1}\left(s_{r}^{n\left(t_{H}\right)}, \alpha_{r}\right)=L H^{-1}\left(T F_{t^{\prime}}^{t}\left(s_{r}^{n\left(t_{H}\right)}, \alpha_{r}\right)\right)$, then it is returned to Case 1 or Case 2.

Example 7. Continuing Example 6, we have

$$
\begin{aligned}
L H(A T, 0) & =\left(s_{15}^{17}, 0\right), \\
L H^{-1}\left(s_{1}^{5}, 0.3\right) & =(N G,-0.4), \\
L H^{-1}\left(s_{10}^{17},-0.4\right) & =L H^{-1}\left(T F_{3}^{4}\left(s_{10}^{17},-0.4\right)\right) \\
& =L H^{-1}\left(s_{5}^{9},-0.2\right)=(A G,-0.2) .
\end{aligned}
$$

2.3. The Dependent Ordered Weighted Average (DOWA) Operator and the Heronian Mean (HM) Operator. The dependent ordered weighted average (DOWA) operator was developed by $\mathrm{Xu}$ [28], it can be defined as follows

Definition 8 (see [28]). Assuming to that $\left(a_{1}, a_{2} \cdots, a_{n}\right)$ is a collection of arguments, $\bar{a}$ is the average value, i.e. $\bar{a}=$ $(1 / n) \sum_{i=1}^{n} a_{i},(\sigma(1), \cdots \sigma(n))$ is a permutation of $(1, \cdots, n)$, such that $a_{\sigma(j)} \geq a_{\sigma(j+1)}$ for all $j=1,2, \cdots, n-1$, if

$$
\begin{aligned}
& \operatorname{DOWA}\left(a_{1}, \cdots, a_{n}\right) \\
& =\sum_{i=1}^{n}\left(\frac{\left(1-\left|a_{\sigma(i)}-\bar{a}\right| / \sum_{i=1}^{n}\left|a_{\sigma(i)}-\bar{a}\right|\right)}{\sum_{i=1}^{n}\left(1-\left|a_{\sigma(i)}-u\right| / \sum_{i=1}^{n}\left|a_{\sigma(i)}-u\right|\right)}\right) a_{\sigma(i)}
\end{aligned}
$$

Then DOWA $\left(a_{1}, a_{2} \cdots, a_{n}\right)$ is called the dependent OWA operator.

The Heronian mean operator has the capacity of capturing the interactions between the input arguments. It can be defined as follows:

Definition 9 (see [32]). Let $x_{i}(i=1, \cdots, n)$ be a collection of non-negative numbers, $E=(0, \infty)$. Then a HM operator of dimension $\mathrm{n}$ is a mapping $H M: E^{n} \longrightarrow E$ which satisfies

$$
H M\left(x_{1}, x_{2}, \cdots x_{n}\right)=\frac{2}{n(n+1)} \sum_{i=1}^{n} \sum_{j=i}^{n} \sqrt{x_{i} x_{j}}
$$

A series of HM operators are provided, such as the generalized HM (GHM) operator and the generalized geometric HM (GGHM) operator.
Definition 10 (see [32]). Let $p \geq 0, q \geq 0, p+q>0$ and $x_{i}(i=1, \cdots, n)$ be a collection of non-negative numbers, $E=$ $(0, \infty)$, then a GHM operator of dimension $\mathrm{n}$ is a mapping $G H M: E^{n} \longrightarrow E$ which satisfies

$$
\begin{aligned}
\operatorname{GHM}^{p, q}\left(x_{1}, x_{2}, \cdots x_{n}\right) \\
=\left(\frac{2}{n(n+1)} \sum_{i=1}^{n} \sum_{j=i}^{n} x_{i}^{p} x_{j}^{q}\right)^{1 /(p+q)}
\end{aligned}
$$

Definition 11 (see [33]). Let $p \geq 0, q \geq 0, p+q>0$ and $x_{i}(i=1, \cdots, n)$ be a collection of non-negative numbers, $E=$ $(0, \infty)$, then a GGHM operator of dimension $\mathrm{n}$ is a mapping $G G H M: E^{n} \longrightarrow E$ which satisfies

$$
\begin{aligned}
& \operatorname{GGHM}^{p, q}\left(x_{1}, x_{2}, \cdots x_{n}\right) \\
& =\frac{1}{p+q} \prod_{i=1}^{n} \prod_{j=i}^{n}\left(p x_{i}+q x_{j}\right)^{2 /(n(n+1))}
\end{aligned}
$$

\section{The IULDWGHM Operator and the IULDWGGHM Operator}

3.1. The IULDWGHM Operator. Inspired by $\mathrm{Xu}$ [28], we will define the intuitionistic unbalanced linguistic term set (IULTS), the intuitionistic unbalanced linguistic number(IULN), then the IULDWGHM operator by combining the dependent operator and the weighted generalized Heronian mean operator will be proposed in the intuitionistic unbalanced linguistic environment.

Definition 12. Let $X=\left\{x_{1}, \cdots, x_{n}\right\}$ be the universe of discourse. An intuitionistic unbalanced linguistic set $U \widetilde{L}$ on $\mathrm{X}$ can be defined as

$$
U \widetilde{L}=\left\{x_{i},\left[U L_{\alpha\left(x_{i}\right)},\left(u_{U \widetilde{L}}\left(x_{i}\right), v_{U \widetilde{L}}\left(x_{i}\right)\right)\right] \mid x_{i} \in X\right\}
$$

where $U L_{\alpha\left(x_{i}\right)}$, belongs to the unbalanced linguistic set, the function $u_{U \widetilde{L}}\left(x_{i}\right)$ and $v_{U \widetilde{L}}\left(x_{i}\right)$ stand for the membership degree and the non-membership degree of $x_{i}$ to $U L_{\alpha\left(x_{i}\right)}$.

Similarly, $\tilde{a}_{i}=\left[U L_{\alpha\left(x_{i}\right)}\left(u_{U \widetilde{L}}\left(x_{i}\right), v_{U \widetilde{L}}\left(x_{i}\right)\right)\right]$ is called an intuitionistic unbalanced linguistic number (IULN).

Definition 13. Given that $\widetilde{a}_{i}=\left[U L_{i},\left(u_{i}, v_{i}\right)\right](i=1,2, \cdots, n)$ is a set of intuitionistic unbalanced linguistic numbers, the mean value of intuitionistic unbalanced linguistic numbers can be defined as

$$
\bar{a}=[U \bar{L},(\bar{u}, \bar{v})]
$$

where $U \bar{L}=L H^{-1}\left(\Delta\left((1 / n) \sum_{i=1}^{n}\left(\Delta^{-1}\left(T_{t_{H}}^{t}\left(L H\left(U L_{i}\right)\right)\right)\right)\right)\right), \bar{u}=$ $1-\left(\prod_{i=1}^{n}\left(1-u_{i}\right)\right)^{1 / n}, \bar{v}=\left(\prod_{i=1}^{n} v_{i}\right)^{1 / n}$.

Definition 14. Assuming that $\widetilde{a}_{i}=\left[U L_{i}\left(u_{i}, v_{i}\right)\right]$ is an intuitionistic unbalanced linguistic number (IULN), then the 
score value and the accurate value of $\tilde{a}_{i}$ can be defined as

$$
\begin{aligned}
& S\left(\tilde{a}_{i}\right)=\frac{\Delta^{-1}\left(T_{t_{H}}^{t}\left(L H\left(U L_{i}\right)\right)\right)}{G\left(t_{H}\right)-1}\left[\frac{1}{2}+\frac{1}{2}\left(u_{i}-v_{i}\right)\right], \\
& H\left(\tilde{a}_{i}\right)=\frac{\Delta^{-1}\left(T_{t_{H}}^{t}\left(L H\left(U L_{i}\right)\right)\right)}{G\left(t_{H}\right)-1}\left(u_{i}+v_{i}\right),
\end{aligned}
$$

where $G\left(t_{H}\right)$ is the granularity of the $t_{H}$ lever in the linguistic hierarchies.

Example 15. Let $\tilde{a}_{1}=[A G,(0.4,0.5)], \tilde{a}_{2}=[M,(0.5,0.5)]$, $\tilde{a}_{3}=[G,(0.3,0.6)]$ be three intuitionistic unbalanced linguistic numbers which the unbalanced linguistic term set with 9 granularity is $\mathrm{S}=\{\mathrm{N}$ (none), $\mathrm{NG}$ (not good), $\mathrm{M}$ (middle), AG(almost good), G(good), QG(quite good), VG(very good), $\mathrm{AT}($ almost total), $\mathrm{T}$ (total) $\}$, the score values and the accurate values of $\widetilde{a}_{1}, \widetilde{a}_{2}, \widetilde{a}_{3}$ are as follows:

$$
\begin{aligned}
S\left(\tilde{a}_{1}\right) & =\frac{\Delta^{-1}\left(T_{4}^{3}(L H(A G))\right)}{17-1}\left[\frac{1}{2}+\frac{1}{2}(0.4-0.5)\right] \\
& =\frac{\Delta^{-1}\left(T_{4}^{3}\left(s_{5}^{9}, 0\right)\right)}{17-1}\left(\frac{1}{2} \cdot 0.9\right)=0.2813 ; \\
H\left(\tilde{a}_{1}\right) & =\frac{\Delta^{-1}\left(T_{4}^{3}(L H(A G))\right)}{17-1}(0.4+0.5) \\
& =\frac{\Delta^{-1}\left(T_{4}^{3}\left(s_{5}^{9}, 0\right)\right)}{17-1} \cdot 0.9=0.1406 ; \\
S\left(\tilde{a}_{2}\right) & =\frac{\Delta^{-1}\left(T_{4}^{2}(L H(M))\right)}{17-1}\left[\frac{1}{2}+\frac{1}{2}(0.5-0.5)\right] \\
& =\frac{\Delta^{-1}\left(T_{4}^{2}\left(s_{2}^{5}, 0\right)\right)}{17-1} \cdot \frac{1}{2}=0.2500 ; \\
H\left(\tilde{a}_{2}\right) & =\frac{\Delta^{-1}\left(T_{4}^{2}(L H(M))\right)}{17-1}(0.5+0.5)
\end{aligned}
$$

$$
\begin{aligned}
& =\frac{\Delta^{-1}\left(T_{4}^{2}\left(s_{2}^{5}, 0\right)\right)}{17-1}=0.5000 \\
S\left(\widetilde{a}_{3}\right) & =\frac{\Delta^{-1}\left(T_{4}^{3}(L H(G))\right)}{17-1}\left[\frac{1}{2}+\frac{1}{2}(0.3-0.6)\right] \\
& =\frac{\Delta^{-1}\left(T_{4}^{3}\left(s_{6}^{9}, 0\right)\right)}{17-1}\left(\frac{1}{2} \cdot 0.7\right)=0.2625 ; \\
H\left(\widetilde{a}_{3}\right) & =\frac{\Delta^{-1}\left(T_{4}^{3}(L H(G))\right)}{17-1}(0.3+0.6) \\
& =\frac{\Delta^{-1}\left(T_{4}^{3}\left(s_{6}^{9}, 0\right)\right)}{17-1} \cdot 0.9=0.6750 .
\end{aligned}
$$

Then we can compare any two IULNs on the basis of score function and accuracy function.

Definition 16. Supposing that $\tilde{a}_{i}=\left[U L_{i},\left(u_{i}, v_{i}\right)\right]$ and $\tilde{a}_{j}=$ $\left[U L_{j},\left(u_{j}, v_{j}\right)\right]$ are any two intuitionistic unbalanced linguistic numbers

(1) If $S\left(\tilde{a}_{i}\right)>S\left(\tilde{a}_{j}\right)$, then $\tilde{a}_{i}>\tilde{a}_{j}$;

(2) If $S\left(\tilde{a}_{i}\right)=S\left(\tilde{a}_{j}\right)$ and $H\left(\tilde{a}_{i}\right)>H\left(\tilde{a}_{j}\right)$, then $\tilde{a}_{i}>\tilde{a}_{j}$.

Definition 17. Let $\tilde{a}_{i}=\left[U L_{i},\left(u_{i}, v_{i}\right)\right]$ and $\tilde{a}_{j}=\left[U L_{j},\left(u_{j}, v_{j}\right)\right]$ be any two intuitionistic unbalanced linguistic numbers, the Hamming distance between $\tilde{a}_{i}$ and $\tilde{a}_{j}$ is

$$
\begin{aligned}
& d\left(\tilde{a}_{i}, \tilde{a}_{j}\right)=\frac{1}{2 G\left(t_{H}\right)-1} \mid\left(1+u_{i}-v_{i}\right) \\
& \cdot \Delta^{-1}\left(T_{t_{H}}^{t}\left(L H\left(U L_{i}\right)\right)\right)-\left(1+u_{j}-v_{j}\right) \\
& \cdot \Delta^{-1}\left(T_{t_{H}}^{t}\left(L H\left(U L_{j}\right)\right)\right) \mid
\end{aligned}
$$

where $G\left(t_{H}\right)$ is the granularity of $t_{H}$ lever in the linguistic hierarchy.

Similarly, the Euclidean distance can be shown as

$$
d\left(\tilde{a}_{i}, \tilde{a}_{j}\right)=\sqrt{\frac{1}{2 G\left(t_{H}\right)-1}\left(\left(1+u_{i}-v_{i}\right) \Delta^{-1}\left(T_{t_{H}}^{t}\left(L H\left(U L_{i}\right)\right)\right)-\left(1+u_{j}-v_{j}\right) \Delta^{-1}\left(T_{t_{H}}^{t}\left(L H\left(U L_{j}\right)\right)\right)\right)^{2}}
$$

Definition 18. Let $\tilde{a}_{1}, \tilde{a}_{2} \cdots, \tilde{a}_{n}$ be a set of intuitionistic unbalanced linguistic numbers, $\bar{a}$ is the arithmetic mean, the similarity between $\widetilde{a}_{i}$ and $\bar{a}$ is

$$
\operatorname{Sim}\left(\tilde{a}_{j}, \bar{a}\right)=1-\frac{d\left(\tilde{a}_{j}, \bar{a}\right)}{\sum_{j=1}^{n} d\left(\tilde{a}_{j}, \bar{a}\right)}
$$

In real life situation, different experts can provide their preference values in the form of the intuitionistic unbalanced linguistic numbers $\left[U L_{1},\left(u_{1}, v_{1}\right)\right], \cdots,\left[U L_{n},\left(u_{n}, v_{n}\right)\right]$. Some experts may assign unduly high preference values to their enjoyed objects while low values to their detested one. The above "false" opinions should be assigned very low weights. In other words, the distance between a preference value and the mean one is lager, the weights should be smaller. Based on Eq. (27), the dependent weight of intuitionistic unbalanced linguistic numbers is

$$
w_{j}=\frac{\operatorname{Sim}\left(\tilde{a}_{j}, \bar{a}\right)}{\sum_{j=1}^{n} \operatorname{Sim}\left(\widetilde{a}_{j}, \bar{a}\right)}
$$


Based on the above dependent weight, we can provide the definition of the intuitionistic unbalanced linguistic dependent weighted generalized Heronian mean (IULDWGHM) operator.

Definition 19. Let $\left(\widetilde{a}_{1}, \widetilde{a}_{2}, \cdots, \widetilde{a}_{n}\right)$ be a set of IULNs and $p, q \geq 0$, IUL be the set of all intuitionistic unbalanced linguistic numbers, an IULDWGHM operator is a mapping $I U L D W G H M: I U L^{n} \longrightarrow I U L$

$$
\begin{aligned}
& I U L D W G H M_{w}^{p, q}\left(\widetilde{a}_{1}, \cdots, \widetilde{a}_{n}\right) \\
& \quad=L H^{-1}\left(\frac { 2 } { n ( n + 1 ) } \left(\bigoplus_{i=1}^{n} \bigoplus_{j=i}^{n}\left(n w_{i}\left(T_{t_{H}}^{t}\left(L H\left(\widetilde{a}_{i}\right)\right)\right)\right)^{p}\right.\right. \\
& \left.\left.\quad \otimes\left(n w_{j}\left(T_{t_{H}}^{t}\left(L H\left(\widetilde{a}_{j}\right)\right)\right)\right)^{q}\right)\right)^{1 /(p+q)}
\end{aligned}
$$

where $w_{i}=\operatorname{Sim}\left(\widetilde{a}_{i}, \bar{a}\right) / \sum_{i=1}^{n} \operatorname{Sim}\left(\widetilde{a}_{i}, \bar{a}\right),(i=1,2, \cdots, n)$

The properties of the IULDWGHM operator can be shown as follows. For convenience, we denote that $s_{b_{j}}=$ $T_{t_{H}}^{t}\left(L H\left(U L_{j}\right)\right)(j=1,2, \cdots, n)$ in the following.

Lemma 20. Let $\left(\widetilde{a}_{1}, \widetilde{a}_{2}, \cdots, \widetilde{a}_{n}\right)$ be a set of IULNs and $p, q \geq 0$, then we have

$$
\begin{aligned}
& \bigoplus_{i=1}^{n}\left(\left(n w_{i}\left(T_{t_{H}}^{t}\left(L H\left(\widetilde{a}_{i}\right)\right)\right)\right)^{p}\right. \\
& \left.\otimes\left(n w_{j}\left(T_{t_{H}}^{t}\left(L H\left(\widetilde{a}_{n}\right)\right)\right)\right)^{q}\right)=\left[s_{\sum_{i=1}^{n}\left(n w_{i} b_{i}\right)^{p} \cdot\left(n w_{n} b_{n}\right)^{q},}\right. \\
& (1
\end{aligned}
$$

$$
\begin{aligned}
& -\prod_{i=1}^{n}\left(1-\left(1-\left(1-u_{i}\right)^{n w_{i}}\right)^{p}\left(1-\left(1-u_{n}\right)^{n w_{n}}\right)^{q}\right), \\
& \left.\left.\prod_{i=1}^{n}\left(1-\left(1-v_{i}^{n w_{i}}\right)^{p}\left(1-v_{n}^{n w_{n}}\right)^{q}\right)\right)\right]
\end{aligned}
$$

Proof. we prove Eq. (29) by means of mathematical induction.

For $n=2$, according to Eqs. (2) - (4), we have

$$
\begin{aligned}
& \bigoplus_{i=1}^{2}\left(\left(2 w_{i}\left(T_{t_{H}}^{t}\left(L H\left(\widetilde{a}_{i}\right)\right)\right)\right)^{p}\right. \\
& \left.\otimes\left(2 w_{2}\left(T_{t_{H}}^{t}\left(L H\left(\widetilde{a}_{2}\right)\right)\right)\right)^{q}\right)=\left[s_{\sum_{i=1}^{2}\left(2 w_{i} b_{i}\right)^{p} \cdot\left(2 w_{2} b_{2}\right)^{q}}\right. \\
& (1 \\
& \quad-\prod_{i=1}^{2}\left(1-\left(1-\left(1-u_{i}\right)^{2 w_{i}}\right)^{p}\left(1-\left(1-u_{2}\right)^{2 w_{2}}\right)^{q}\right), \\
& \left.\left.\prod_{i=1}^{2}\left(1-\left(1-v_{i}^{2 w_{i}}\right)^{p}\left(1-v_{2}^{2 w_{2}}\right)^{q}\right)\right)\right] .
\end{aligned}
$$

Therefore, Eq. (29) holds for $\mathrm{n}=2$.

If Eq. (29) holds for $n=k$,

Then when $n=k+1$, we can obtain

$$
\begin{aligned}
& \bigoplus_{i=1}^{k+1}\left(\left((k+1) w_{i}\left(T_{t_{H}}^{t}\left(L H\left(\widetilde{a}_{i}\right)\right)\right)\right)^{p} \otimes\left((k+1) w_{k+1}\left(T_{t_{H}}^{t}\left(L H\left(\widetilde{a}_{k+1}\right)\right)\right)\right)^{q}\right)=\bigoplus_{i=1}^{k}\left(\left((k+1) w_{i} s_{b_{i}}\right)^{p} \otimes\left((k+1) w_{k+1} s_{b_{k+1}}\right)^{q}\right) \\
& \oplus\left(\left((k+1) w_{k+1} s_{b_{k+1}}\right)^{p} \otimes\left((k+1) w_{k+1} s_{b_{k+1}}\right)^{q}\right) \\
& =\left[s_{\sum_{i=1}^{k+1}\left((k+1) w_{i} b_{i}\right)^{p} \cdot\left((k+1) w_{k+1} b_{k+1}\right)^{q}}\left(\begin{array}{c}
1-\prod_{i=1}^{k+1}\left(1-\left(1-\left(1-u_{i}\right)^{(k+1) w_{i}}\right)^{p}\left(1-\left(1-u_{k+1}\right)^{(k+1) w_{(k+1)}}\right)^{q}\right), \\
\prod_{i=1}^{k+1}\left(1-\left(1-v_{i}^{(k+1) w_{i}}\right)^{p}\left(1-v_{k+1}^{(k+1) w_{k+1}}\right)^{q}\right)
\end{array}\right)\right] \text {, }
\end{aligned}
$$

which means that Eq. (29) also holds for $n=k+1$. We complete the proof.
Theorem 21. Let $\left(\widetilde{a}_{1}, \widetilde{a}_{2}, \cdots, \widetilde{a}_{n}\right)$ be a set of IULNs and $p, q \geq$ 0 , the aggregated result of Eq. (28) is still an IULN and

$$
\begin{aligned}
& \operatorname{IULDWGHM} M_{w}^{p, q}\left(\widetilde{a}_{1}, \cdots, \widetilde{a}_{n}\right) \\
& \left.\qquad \begin{array}{c}
\operatorname{LH}^{-1}\left(s_{\alpha}\right),\left(1-\left(\prod_{i=1}^{n} \prod_{j=i}^{n}\left(1-\left(1-\left(1-u_{i}\right)^{n w_{i}}\right)^{p}\left(1-\left(1-u_{j}\right)^{n w_{j}}\right)^{q}\right)\right)^{2 / n(n+1)}\right)^{1 /(p+q)} \\
1-\left(1-\left(\prod_{i=1}^{n} \prod_{j=i}^{n}\left(1-\left(1-v_{i}^{n w_{i}}\right)^{p}\left(1-v_{j}^{n w_{j}}\right)^{q}\right)\right)^{2 / n(n+1)}\right)^{1 /(p+q)}
\end{array}\right)
\end{aligned}
$$


Where

$$
\begin{gathered}
\alpha=\left(\frac{2}{n(n+1)} \sum_{i=1}^{n} \sum_{j=i}^{n}\left(n w_{i}\left(\Delta^{-1}\left(T_{t_{H}}^{t}\left(L H\left(a_{i}\right)\right)\right)\right)\right)^{p}\right. \\
\left.\cdot\left(n w_{j}\left(\Delta^{-1}\left(T_{t_{H}}^{t}\left(L H\left(a_{j}\right)\right)\right)\right)\right)^{q}\right)^{1 /(p+q)} .
\end{gathered}
$$

Proof. by Eq. (4) and (5), we can have

$$
\begin{aligned}
& \left(n \cdot w_{i} \cdot\left(T_{t_{H}}^{t}\left(L H\left(\tilde{a}_{i}\right)\right)\right)\right)^{p} \\
& =\left[s_{\left(n w_{i} b_{i}\right)^{p}},\left(1-\left(1-u_{i}\right)^{n w_{i}}\right)^{p}, 1-\left(1-v_{i}^{n w_{i}}\right)^{p}\right] ; \\
& \left(n \cdot w_{j} \cdot\left(T_{t_{H}}^{t}\left(L H\left(\tilde{a}_{j}\right)\right)\right)\right)^{q} \\
& =\left[s_{\left(n w_{j} b_{j}\right)^{q}},\left(1-\left(1-u_{j}\right)^{n w_{j}}\right)^{q}, 1-\left(1-v_{j}^{n w_{j}}\right)^{q}\right] ; \\
& \left(n \cdot w_{i} \cdot\left(T_{t_{H}}^{t}\left(L H\left(\widetilde{a}_{i}\right)\right)\right)\right)^{p} \otimes\left(n \cdot w_{j}\right. \\
& \left.\cdot\left(T_{t_{H}}^{t}\left(L H\left(\tilde{a}_{j}\right)\right)\right)\right)^{q}
\end{aligned}
$$

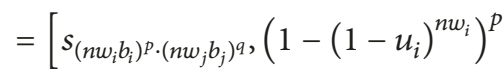

$$
\begin{aligned}
& \left.\cdot\left(1-\left(1-u_{j}\right)^{n w_{j}}\right)^{q}, 1-\left(1-v_{i}^{n w_{i}}\right)^{p}\left(1-v_{j}^{n w_{j}}\right)^{q}\right]
\end{aligned}
$$

In the following, we need to prove following Eq. (35) by mathematical induction on $\mathrm{n}$.

$$
\begin{aligned}
& \bigoplus_{i=1}^{n} \bigoplus_{j=i}^{n}\left(n \cdot w_{i} \cdot\left(T_{t_{H}}^{t}\left(L H\left(\tilde{a}_{i}\right)\right)\right)\right)^{p} \otimes\left(n \cdot w_{j}\right. \\
& \left.\cdot\left(T_{t_{H}}^{t}\left(L H\left(\tilde{a}_{j}\right)\right)\right)\right)^{q}=\left[s_{\sum_{i=1}^{n} \sum_{j=i}^{n}\left(n w_{i} b_{i}\right)^{p} \cdot\left(n w_{j} b_{j}\right)^{q},(1}\right. \\
& -\prod_{i=1}^{n} \prod_{j=i}^{n}\left(1-\left(1-\left(1-u_{i}\right)^{n w_{i}}\right)^{p}\left(1-\left(1-u_{j}\right)^{n w_{j}}\right)^{q}\right), \\
& \left.\left.\prod_{i=1}^{n} \prod_{j=i}^{n}\left(1-\left(1-v_{i}^{n w_{i}}\right)^{p}\left(1-v_{j}^{n w_{j}}\right)^{q}\right)\right)\right]
\end{aligned}
$$

For $\mathrm{n}=2$,

$$
\begin{aligned}
\bigoplus_{i=1}^{2} \bigoplus_{j=i}^{2}\left(2 \cdot w_{i} \cdot\left(T_{t_{H}}^{t}\left(L H\left(\tilde{a}_{i}\right)\right)\right)\right)^{p} \otimes\left(2 \cdot w_{j}\right. \\
\left.\cdot\left(T_{t_{H}}^{t}\left(L H\left(\tilde{a}_{j}\right)\right)\right)\right)^{q}=\left(\left(2 w_{1} b_{1}\right)^{p} \cdot\left(2 w_{1} b_{1}\right)^{q}\right) \\
\oplus\left(\left(2 w_{1} b_{1}\right)^{p} \cdot\left(2 w_{2} b_{2}\right)^{q}\right) \oplus\left(\left(2 w_{2} b_{2}\right)^{p} \cdot\left(2 w_{2} b_{2}\right)^{q}\right) \\
=\left[s_{\sum_{i=1}^{2} \sum_{j=i}^{2}\left(2 w_{i} b_{i}\right)^{p} \cdot\left(2 w_{j} b_{j}\right)^{q},(1}\right. \\
-\prod_{i=1}^{2} \prod_{j=i}^{2}\left(1-\left(1-\left(1-u_{i}\right)^{2 w_{i}}\right)^{p}\left(1-\left(1-u_{j}\right)^{2 w_{j}}\right)^{q}\right), \\
\left.\left.\prod_{i=1}^{2} \prod_{j=i}^{2}\left(1-\left(1-v_{i}^{2 w_{i}}\right)^{p}\left(1-v_{j}^{2 w_{j}}\right)^{q}\right)\right)\right]
\end{aligned}
$$

If Eq.(35) for $n=k$, i.e.,

$$
\begin{aligned}
& \bigoplus_{i=1}^{k} \bigoplus_{j=i}^{k}\left(k \cdot w_{i} \cdot\left(T_{t_{H}}^{t}\left(L H\left(\tilde{a}_{i}\right)\right)\right)\right)^{p} \otimes\left(k \cdot w_{j}\right. \\
& \left.\cdot\left(T_{t_{H}}^{t}\left(L H\left(\tilde{a}_{j}\right)\right)\right)\right)^{q}=\left[s_{\sum_{i=1}^{k} \sum_{j=i}^{k}\left(k w_{i} b_{i}\right){ }^{p} \cdot\left(k w_{j} b_{j}\right)^{q}},(1\right. \\
& \quad-\prod_{i=1}^{k} \prod_{j=i}^{k}\left(1-\left(1-\left(1-u_{i}\right)^{k w_{i}}\right)^{p}\left(1-\left(1-u_{j}\right)^{k w_{j}}\right)^{q}\right), \\
& \left.\left.\prod_{i=1}^{k} \prod_{j=i}^{k}\left(1-\left(1-v_{i}^{k w_{i}}\right)^{p}\left(1-v_{j}^{k w_{j}}\right)^{q}\right)\right)\right]
\end{aligned}
$$

Then, for $\mathrm{n}=\mathrm{k}+1$, we obtain

$$
\begin{aligned}
& \bigoplus_{i=1}^{k+1} \bigoplus_{j=i}^{k+1}\left((k+1) \cdot w_{i} \cdot\left(T_{t_{H}}^{t}\left(L H\left(\tilde{a}_{i}\right)\right)\right)\right)^{p} \\
& \otimes\left((k+1) \cdot w_{j} \cdot\left(T_{t_{H}}^{t}\left(L H\left(\tilde{a}_{j}\right)\right)\right)\right)^{q} \\
& =\bigoplus_{i=1}^{k} \bigoplus_{j=i}^{k}\left(\left((k+1) w_{i} \tilde{a}_{i}\right)^{p} \otimes\left((k+1) w_{j} \tilde{a}_{j}\right)^{q}\right) \\
& \oplus\left(\bigoplus_{i=1}^{k+1}\left(\left((k+1) w_{i} \tilde{a}_{i}\right)^{p} \otimes\left((k+1) w_{k+1} \tilde{a}_{k+1}\right)^{q}\right)\right)
\end{aligned}
$$

By Eq. (29), (37), we can transform (38) as

$$
\begin{aligned}
& \bigoplus_{i=1}^{k+1} \bigoplus_{j=i}^{k+1}\left((k+1) \cdot w_{i} \cdot\left(T_{t_{H}}^{t}\left(L H\left(\tilde{a}_{i}\right)\right)\right)\right)^{p} \otimes\left((k+1) \cdot w_{j} \cdot\left(T_{t_{H}}^{t}\left(L H\left(\tilde{a}_{j}\right)\right)\right)\right)^{q} \\
& =\left[\left(1-\prod_{i=1}^{k+1} \prod_{j=i}^{k+1}\left(1-\left(1-\left(1-u_{i}\right)^{(k+1) w_{i}}\right)^{p}\left(1-\left(1-u_{j}\right)^{(k+1) w_{j}}\right)^{q}\right), \prod_{i=1}^{s_{i=1}^{k+1} \sum_{j=i}^{k+1}\left((k+1) w_{i} b_{i}\right)^{p} \cdot\left((k+1) w_{j} b_{j}\right)^{q}},\right.\right.
\end{aligned}
$$


Mathematical Problems in Engineering

9

Furthermore, we have

$$
\begin{aligned}
& \frac{2}{n(n+1)}\left(\bigoplus_{i=1}^{n} \bigoplus_{j=i}^{n}\left(n \cdot w_{i} \cdot\left(T_{t_{H}}^{t}\left(L H\left(\widetilde{a}_{i}\right)\right)\right)\right)^{p} \otimes\left(n \cdot w_{j} \cdot\left(T_{t_{H}}^{t}\left(L H\left(\widetilde{a}_{j}\right)\right)\right)\right)^{q}\right) \\
& =\left[s_{(2 / n(n+1))} \sum_{i=1}^{n} \sum_{j=i}^{n}\left(n w_{i} b_{i}\right)^{p} \cdot\left(n w_{j} b_{j}\right)^{q},\left(1-\left(\prod_{i=1}^{n} \prod_{j=i}^{n}\left(1-\left(1-\left(1-u_{i}\right)^{n w_{i}}\right)^{p}\left(1-\left(1-u_{j}\right)^{n w_{j}}\right)^{q}\right)\right)^{2 / n(n+1)},\left(\prod_{i=1}^{n} \prod_{j=i}^{n}\left(1-\left(1-v_{i}^{n w_{i}}\right)^{p}\left(1-v_{j}^{n w_{j}}\right)^{q}\right)\right)^{2 / n(n+1)}\right)\right] \\
& \left(\frac{2}{n(n+1)}\left(\bigoplus_{i=1}^{n} \bigoplus_{j=i}^{n}\left(n w_{i}\left(T_{t_{H}}^{t}\left(L H\left(\widetilde{a}_{i}\right)\right)\right)\right)^{p} \otimes\left(n w_{j}\left(T_{t_{H}}^{t}\left(L H\left(\widetilde{a}_{j}\right)\right)\right)\right)^{q}\right)\right)^{1 /(p+q)} \\
& \left.=\left[\left(\left(1-\left(\prod_{i=1}^{n} \prod_{j=i}^{n}\left(1-\left(1-\left(1-u_{i}\right)^{n w_{i}}\right)^{p}\left(1-\left(1-u_{j}\right)^{n w_{j}}\right)^{q}\right)\right)^{2 / n(n+1)}\right)^{1 /(p+q)}, 1-\left(1-\left(\left(\prod_{i=1}^{n} \prod_{j=i}^{n}\left(1-\left(1-v_{i}^{n w_{i}}\right)^{p}\left(1-v_{j}^{n w_{j}}\right)^{q}\right)\right)^{2 / n(n+1)}\right)\right)^{1 /(p+q)}\right)\right)\right]
\end{aligned}
$$

Thus,

$$
\left.\begin{array}{rl}
L H^{-1} & \left.\frac{2}{n(n+1)}\left(\bigoplus_{i=1}^{n} \bigoplus_{j=i}^{n}\left(n w_{i}\left(T_{t_{H}}^{t}\left(L H\left(\tilde{a}_{i}\right)\right)\right)\right)^{p} \otimes\left(n w_{j}\left(T_{t_{H}}^{t}\left(L H\left(\tilde{a}_{j}\right)\right)\right)\right)^{q}\right)\right)^{1 /(p+q)} \\
= & \left(\begin{array}{c}
L H^{-1}\left(s_{\alpha}\right),\left(1-\left(\prod_{i=1}^{n} \prod_{j=i}^{n}\left(1-\left(1-\left(1-u_{i}\right)^{n w_{i}}\right)^{p}\left(1-\left(1-u_{j}\right)^{n w_{j}}\right)^{q}\right)\right)^{2 / n(n+1)}\right)^{1 /(p+q)} \\
1-\left(1-\left(\prod_{i=1}^{n} \prod_{j=i}^{n}\left(1-\left(1-v_{i}^{n w_{i}}\right)^{p}\left(1-v_{j}^{n w_{j}}\right)^{q}\right)\right)^{2 / n(n+1)}\right)^{1 /(p+q)}
\end{array}\right)
\end{array}\right)
$$

where

$$
\begin{gathered}
\alpha=\left(\frac{2}{n(n+1)} \sum_{i=1}^{n} \sum_{j=i}^{n}\left(n w_{i}\left(\Delta^{-1}\left(T_{t_{H}}^{t}\left(L H\left(a_{i}\right)\right)\right)\right)\right)^{p}\right. \\
\left.\cdot\left(n w_{j}\left(\Delta^{-1}\left(T_{t_{H}}^{t}\left(L H\left(a_{j}\right)\right)\right)\right)\right)^{q}\right)^{1 /(p+q)} .
\end{gathered}
$$

Next, we prove that the sum of the membership degree and the non-membership degree belongs to $[0,1]$.

Since

$$
\begin{aligned}
& 0 \leq\left(1-\left(\prod_{i=1}^{n} \prod_{j=i}^{n}\left(1-\left(1-\left(1-u_{i}\right)^{n w_{i}}\right)^{p}\left(1-\left(1-u_{j}\right)^{n w_{j}}\right)^{q}\right)\right)^{2 / n(n+1)}\right)^{1 /(p+q)} \leq 1, \\
& 0 \leq 1-\left(1-\left(\prod_{i=1}^{n} \prod_{j=i}^{n}\left(1-\left(1-v_{i}^{n w_{i}}\right)^{p}\left(1-v_{j}^{n w_{j}}\right)^{q}\right)\right)^{2 / n(n+1)}\right)^{1 /(p+q)} \leq 1, \quad u_{i} \leq 1-v_{i} \forall i=1,2, \cdots, n,
\end{aligned}
$$


Thus

$$
\begin{aligned}
& \left(1-\left(\prod_{i=1}^{n} \prod_{j=i}^{n}\left(1-\left(1-\left(1-u_{i}\right)^{n w_{i}}\right)^{p}\left(1-\left(1-u_{j}\right)^{n w_{j}}\right)^{q}\right)\right)^{2 / n(n+1)}\right)^{1 /(p+q)}+1 \\
& -\left(1-\left(\prod_{i=1}^{n} \prod_{j=i}^{n}\left(1-\left(1-v_{i}^{n w_{i}}\right)^{p}\left(1-v_{j}^{n w_{j}}\right)^{q}\right)\right)^{2 / n(n+1)}\right)^{1 /(p+q)} \\
& \quad \leq\left(1-\left(\prod_{i=1}^{n} \prod_{j=i}^{n}\left(1-\left(1-v_{i}^{n w_{i}}\right)^{p}\left(1-v_{j}^{n w_{j}}\right)^{q}\right)\right)^{2 / n(n+1)}\right)^{1 /(p+q)}+1 \\
& \quad-\left(1-\left(\prod_{i=1}^{n} \prod_{j=i}^{n}\left(1-\left(1-v_{i}^{n w_{i}}\right)^{p}\left(1-v_{j}^{n w_{j}}\right)^{q}\right)\right)^{2 / n(n+1)}\right)^{1 /(p+q)}=1 .
\end{aligned}
$$

This completes the proof of Theorem 21.

Theorem 22 (idempotency). If $\widetilde{a}_{i}=\widetilde{a}$ for all $i=1, \cdots, n$, then

$$
\begin{aligned}
& \operatorname{IULDWGHM}_{w}^{p, q}\left(\widetilde{a}_{1}, \cdots, \widetilde{a}_{n}\right) \\
& \quad=\operatorname{IULDWGHM} M_{w}^{p, q}(\widetilde{a}, \cdots, \widetilde{a})=\widetilde{a}
\end{aligned}
$$

$$
\begin{aligned}
& \operatorname{IULDWGHM} M_{w}^{p, q}\left(\widetilde{a}_{1}, \cdots, \widetilde{a}_{n}\right) \\
& =L H^{-1}\left(\frac{2}{n(n+1)}\left(\bigoplus_{i=1}^{n} \bigoplus_{j=i}^{n}\left(n \cdot w_{i} \cdot\left(T_{t_{H}}^{t}\left(L H\left(\widetilde{a}_{i}\right)\right)\right)\right)^{p} \otimes\left(n \cdot w_{j} \cdot\left(T_{t_{H}}^{t}\left(L H\left(\widetilde{a}_{j}\right)\right)\right)\right)^{q}\right)\right)^{1 /(p+q)} \\
& =L H^{-1}\left(\left(\bigoplus_{i=1}^{n} \bigoplus_{j=i}^{n}\left(n \cdot \frac{1}{n}\left(T_{t_{H}}^{t}(L H(\widetilde{a}))\right)\right)^{p} \otimes\left(n \cdot \frac{1}{n}\left(T_{t_{H}}^{t}(L H(\widetilde{a}))\right)\right)^{q}\right)\right)^{1 /(p+q)} \\
& =L H^{-1}\left(\frac{2}{n(n+1)}\left(\bigoplus_{i=1}^{n} \bigoplus_{j=i}^{n}\left(\left(\left(T_{t_{H}}^{t}(L H(\widetilde{a}))\right)\right)^{p+q}\right)\right)\right)^{1 /(p+q)}=L H^{-1}\left(\left(\left(\left(\left(T_{t_{H}}^{t}(L H(\widetilde{a}))\right)\right)^{p+q}\right)\right)^{1 /(p+q)}\right)=\widetilde{a} .
\end{aligned}
$$

Theorem 23 (monotonicity). Let $\left.\widetilde{\alpha}_{i}=\left[U L_{\alpha_{i}}, u_{\alpha_{i}}, v_{\alpha_{i}}\right)\right]$ and $\left.\widetilde{\beta}_{i}=\left[U L_{\beta_{i}}, u_{\beta_{i}}, v_{\beta_{i}}\right)\right]$ be any two collections of IULNs, if

$$
u_{\alpha_{i}} \leq u_{\beta_{i}}, v_{\alpha_{i}} \geq v_{\beta_{i}}, U L_{\alpha_{i}} \leq U L_{\beta_{i}} \text { for all } i=1, \cdots, n \text {, }
$$
then

$$
\begin{aligned}
& \operatorname{IULDWGHM} M_{w}^{p, q}\left(\widetilde{\alpha}_{1}, \cdots, \widetilde{\alpha}_{n}\right) \\
& \quad \leq \operatorname{IULDWGHM} M_{w}^{p, q}\left(\widetilde{\beta}_{1}, \cdots, \widetilde{\beta}_{n}\right)
\end{aligned}
$$

Proof. Since $u_{\alpha_{i}} \leq u_{\beta_{i}}, v_{\alpha_{i}} \geq v_{\beta_{i}}, U L_{\alpha_{i}} \leq U L_{\beta_{i}}$ for all $i$, then we have

$$
\begin{gathered}
1-\left(\prod_{i=1}^{n} \prod_{j=i}^{n}\left(1-\left(1-\left(1-u_{\alpha_{i}}\right)^{n w_{i}}\right)^{p}\left(1-\left(1-u_{\alpha_{j}}\right)^{n w_{j}}\right)^{q}\right)\right)^{2 / n(n+1)} \leq 1 \\
-\left(\prod_{i=1}^{n} \prod_{j=i}^{n}\left(1-\left(1-\left(1-u_{\beta_{i}}\right)^{n w_{i}}\right)^{p}\left(1-\left(1-u_{\beta_{j}}\right)^{n w_{j}}\right)^{q}\right)\right)^{2 / n(n+1)},
\end{gathered}
$$




$$
\begin{aligned}
& \left(\prod_{i=1}^{n} \prod_{j=i}^{n}\left(1-\left(1-v_{\alpha_{i}}^{n w_{i}}\right)^{p}\left(1-v_{\alpha_{j}}^{n w_{j}}\right)^{q}\right)\right)^{2 / n(n+1)} \geq\left(\prod_{i=1}^{n} \prod_{j=i}^{n}\left(1-\left(1-v_{\beta_{i}}^{n w_{i}}\right)^{p}\left(1-v_{\beta_{j}}^{n w_{j}}\right)^{q}\right)\right)^{2 / n(n+1)}, \\
& \left(\frac{2}{n(n+1)} \sum_{i=1}^{n} \sum_{j=i}^{n}\left(n w_{i} b_{\alpha_{i}}\right)^{p} \cdot\left(n w_{j} b_{\alpha_{j}}\right)^{q}\right)^{p+q} \leq\left(\frac{2}{n(n+1)} \sum_{i=1}^{n} \sum_{j=i}^{n}\left(n w_{i} b_{\beta_{i}}\right)^{p} \cdot\left(n w_{j} b_{\beta_{j}}\right)^{q}\right)^{p+q},
\end{aligned}
$$

\section{Thus}

$$
\begin{aligned}
& \left(\frac{2}{n(n+1)} \sum_{i=1}^{n} \sum_{j=i}^{n}\left(n w_{i} b_{\alpha_{i}}\right)^{p} \cdot\left(n w_{j} b_{\alpha_{j}}\right)^{q}\right)^{p+q}
\end{aligned}
$$

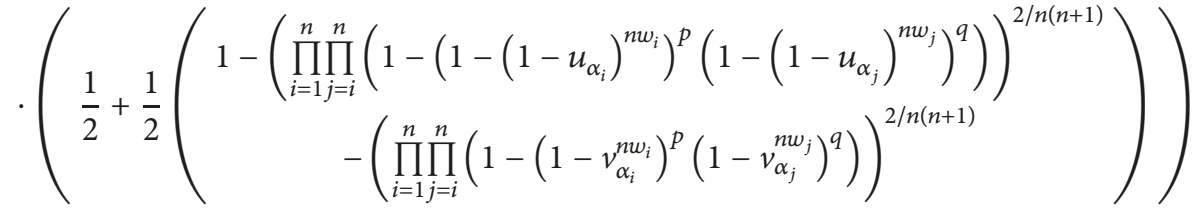

$$
\begin{aligned}
& \leq\left(\frac{2}{n(n+1)} \sum_{i=1}^{n} \sum_{j=i}^{n}\left(n w_{i} b_{\beta_{i}}\right)^{p} \cdot\left(n w_{j} b_{\beta_{j}}\right)^{q}\right)^{p+q} \\
& \left(\frac{1}{2}+\frac{1}{2}\left(\begin{array}{c}
1-\left(\prod_{i=1}^{n} \prod_{j=i}^{n}\left(1-\left(1-\left(1-u_{\beta_{i}}\right)^{n w_{i}}\right)^{p}\left(1-\left(1-u_{\beta_{j}}\right)^{n w_{j}}\right)^{q}\right)\right)^{2 / n(n+1)} \\
-\left(\prod_{i=1}^{n} \prod_{j=i}^{n}\left(1-\left(1-v_{\beta_{i}}^{n w_{i}}\right)^{p}\left(1-v_{\beta_{j}}^{n w_{j}}\right)^{q}\right)\right)^{2 / n(n+1)}
\end{array}\right)\right)
\end{aligned}
$$

i.e. $\operatorname{IULDWGHM} M_{w}^{p, q}\left(\widetilde{\alpha}_{1}, \cdots, \widetilde{\alpha}_{n}\right) \leq \operatorname{IULDWGHM} M_{w}^{p, q}\left(\widetilde{\beta}_{1}\right.$, $\left.\cdots, \widetilde{\beta}_{n}\right)$

Theorem 24 (boundedness). The IULDWGHM operator lies between the maximum operator and the minimum operator, i.e.

$$
\begin{aligned}
\widetilde{\alpha}_{*} & =\min \left(\widetilde{\alpha}_{1}, \cdots, \widetilde{\alpha}_{n}\right) \\
& \leq \operatorname{IULDWGHM} M_{w}^{p, q}\left(\widetilde{\alpha}_{1}, \cdots, \widetilde{\alpha}_{n}\right) \\
& \leq \max \left(\widetilde{\alpha}_{1}, \cdots, \widetilde{\alpha}_{n}\right)=\widetilde{\alpha}^{*}
\end{aligned}
$$

Proof. Since $\widetilde{\alpha}_{*} \leq \widetilde{\alpha}_{j} \leq \widetilde{\alpha}^{*}$, then we have

$$
\begin{aligned}
& L H^{-1}\left(\frac { 2 } { n ( n + 1 ) } \left(\bigoplus_{i=1}^{n} \bigoplus_{j=i}^{n}\left(n w_{i}\left(T_{t_{H}}^{t}\left(L H\left(\widetilde{a}_{*}\right)\right)\right)\right)^{p}\right.\right. \\
& \left.\left.\quad \otimes\left(n w_{j}\left(T_{t_{H}}^{t}\left(L H\left(\widetilde{a}_{*}\right)\right)\right)\right)^{q}\right)\right)^{1 /(p+q)} \\
& \quad \leq L H^{-1}\left(\frac { 2 } { n ( n + 1 ) } \left(\bigoplus_{i=1}^{n} \bigoplus_{j=i}^{n}\left(n w_{i}\left(T_{t_{H}}^{t}\left(L H\left(\widetilde{a}_{i}\right)\right)\right)\right)^{p}\right.\right.
\end{aligned}
$$

$$
\begin{aligned}
& \left.\left.\otimes\left(n w_{j}\left(T_{t_{H}}^{t}\left(L H\left(\widetilde{a}_{j}\right)\right)\right)\right)^{q}\right)\right)^{1 /(p+q)} \\
& \leq L H^{-1}\left(\frac { 2 } { n ( n + 1 ) } \left(\bigoplus_{i=1}^{n} \bigoplus_{j=i}^{n}\left(n w_{i}\left(T_{t_{H}}^{t}\left(L H\left(\widetilde{a}^{*}\right)\right)\right)\right)^{p}\right.\right. \\
& \left.\left.\otimes\left(n w_{j}\left(T_{t_{H}}^{t}\left(L H\left(\widetilde{a}^{*}\right)\right)\right)\right)^{q}\right)\right)^{1 /(p+q)}
\end{aligned}
$$

Thus $\widetilde{\alpha}_{*}=\min \left(\widetilde{\alpha}_{1}, \cdots, \widetilde{\alpha}_{n}\right) \leq \operatorname{IULDWGHM} M_{w}^{p, q}\left(\widetilde{\alpha}_{1}, \cdots\right.$, $\left.\tilde{\alpha}_{n}\right) \leq \max \left(\widetilde{\alpha}_{1}, \cdots, \widetilde{\alpha}_{n}\right)=\tilde{\alpha}^{*}$.

Based on the different values of the parameters $p$ and $q$, we can derive the following special cases of IULDWGHM.

Case 4. If $\mathrm{q}=0$, the IULDWGHM operator degenerates to IUL generalized weighted mean operator.

$$
\begin{gathered}
\operatorname{IULDWGHM} M_{w}^{p, 0}\left(\tilde{a}_{1}, \cdots, \tilde{a}_{n}\right)=L H^{-1}\left(\frac{1}{n}\right. \\
\left.\cdot \bigoplus_{i=1}^{n}\left(n \cdot w_{i} \cdot\left(T_{t_{H}}^{t}\left(L H\left(\tilde{a}_{i}\right)\right)\right)\right)^{p}\right)^{1 / p}
\end{gathered}
$$




$$
\begin{aligned}
& =\left(L H^{-1}\left(s_{\left((1 / n) \sum_{i=1}^{n}\left(n \cdot w_{i} \cdot\left(\Delta^{-1}\left(T_{t_{H}}^{t}\left(L H\left(a_{i}\right)\right)\right)\right)\right)^{p}\right)^{1 / p}}\right),\right. \\
& \left(1-\prod_{i=1}^{n}\left(1-\left(1-\left(1-u_{i}\right)^{n w_{i}}\right)^{p}\right)^{1 / n}\right)^{1 / p}, 1 \\
& \left.-\left(1-\prod_{i=1}^{n}\left(1-\left(1-v_{j}^{n w_{j}}\right)^{p}\right)^{1 / n}\right)^{1 / p}\right)
\end{aligned}
$$

Case 5. If $\mathrm{p}=1, \mathrm{q}=0$, the IULDWGHM operator degenerates to IUL arithmetic weighted mean operator.

$$
\begin{aligned}
& \operatorname{IULDWGHM} M_{w}^{1,0}\left(\widetilde{a}_{1}, \cdots, \widetilde{a}_{n}\right)=L H^{-1}\left(\frac{1}{n}\right. \\
& \left.\cdot \bigoplus_{i=1}^{n}\left(n \cdot w_{i} \cdot\left(T_{t_{H}}^{t}\left(L H\left(\widetilde{a}_{i}\right)\right)\right)\right)\right) \\
& =\left(L H^{-1}\left(s_{\sum_{i=1}^{n}\left(w_{i} \cdot\left(\Delta^{-1}\left(T_{t_{H}}^{t}\left(L H\left(a_{i}\right)\right)\right)\right)\right)}\right)\right.
\end{aligned}
$$

$$
\left.\left(1-\prod_{i=1}^{n}\left(1-u_{i}\right)^{w_{i}}, \prod_{i=1}^{n} v_{j}^{w_{j}}\right)\right)
$$

\subsection{The IULDWGGHM Operator}

Definition 25. Let $\left(\widetilde{a}_{1}, \widetilde{a}_{2}, \cdots, \widetilde{a}_{n}\right)$ be a set of IULNs and $p, q \geq 0$, IUL is the set of all intuitionistic unbalanced linguistic numbers, an IULDWGGHM operator is a mapping $I U L D W G G H M: I U L^{n} \longrightarrow I U L$

$$
\begin{aligned}
& \text { IULDWGGHM } M_{w}^{p, q}\left(\widetilde{a}_{1}, \cdots, \widetilde{a}_{n}\right) \\
& \quad=L H^{-1}\left(\frac { 1 } { p + q } \left(\bigotimes _ { i = 1 } ^ { n } \bigotimes _ { j = i } ^ { n } \left(p\left(T_{t_{H}}^{t}\left(L H\left(\widetilde{a}_{i}\right)\right)^{n w_{i}}\right)\right.\right.\right. \\
& \left.\left.\left.\quad \oplus q\left(\left(T_{t_{H}}^{t}\left(L H\left(\widetilde{a}_{j}\right)\right)\right)^{n w_{j}}\right)\right)^{q}\right)^{2 / n(n+1)}\right)
\end{aligned}
$$

Lemma 26. Let $\left(\widetilde{a}_{1}, \widetilde{a}_{2}, \cdots, \widetilde{a}_{n}\right)$ be a set of IULNs and $p, q \geq 0$, then we have

$$
\begin{aligned}
& \bigotimes_{i=1}^{n}\left(\left(p\left(T_{t_{H}}^{t}\left(L H\left(\widetilde{a}_{i}\right)\right)\right)\right)^{n w_{i}} \oplus\left(q\left(T_{t_{H}}^{t}\left(L H\left(\widetilde{a}_{n}\right)\right)\right)\right)^{n w_{j}}\right) \\
& \quad=\left[s_{\prod_{i=1}^{n}\left(p b_{i}^{n w_{i}}+q b_{n}^{n w_{n}}\right)},\left(\prod_{i=1}^{n}\left(1-\left(1-u_{i}^{n w_{i}}\right)^{p}\left(1-u_{n}^{n w_{n}}\right)^{q}, 1-\prod_{i=1}^{n}\left(1-\left(1-\left(1-v_{i}\right)^{n w_{i}}\right)^{p}\left(1-\left(1-v_{n}\right)^{n w_{n}}\right)^{q}\right)\right)\right)\right]
\end{aligned}
$$

The proof process of Lemma 26 is shown in Appendix.

Theorem 27. Let $\left(\widetilde{a}_{1}, \widetilde{a}_{2}, \cdots, \widetilde{a}_{n}\right)$ be a set of IULNs and $p, q \geq$ 0 , the aggregated result of Eq. (54) is still an IULN and

$$
\operatorname{IULDWGGHM} M_{w}^{p, q}\left(\widetilde{a}_{1}, \cdots, \widetilde{a}_{n}\right)=\left(\begin{array}{l}
\operatorname{LH}^{-1}\left(s_{\alpha^{\prime}}\right), 1-\left(1-\prod_{i=1}^{n} \prod_{j=i}^{n}\left(1-\left(1-u_{i}^{n w_{i}}\right)^{p}\left(1-u_{j}^{n w_{j}}\right)^{q}\right)^{2 / n(n+1)}\right)^{1 /(p+q)}, \\
\left(1-\left(\prod_{i=1}^{n} \prod_{j=i}^{n}\left(1-\left(1-\left(1-v_{i}\right)^{n w_{i}}\right)^{p}\left(1-\left(1-v_{j}\right)^{n w_{j}}\right)^{q}\right)\right)^{2 / n(n+1)}\right)^{1 /(p+q)}
\end{array}\right)
$$

where

$$
\alpha^{\prime}=\frac{1}{p+q} \prod_{i=1}^{n} \prod_{j=i}^{n}\left(p\left(\Delta^{-1}\left(T_{t_{H}}^{t}\left(L H\left(a_{i}\right)\right)\right)\right)^{n w_{i}}+q\left(\Delta^{-1}\left(T_{t_{H}}^{t}\left(L H\left(a_{j}\right)\right)\right)\right)^{n w_{j}}\right)^{2 / n(n+1)} .
$$


The proof process of Theorem 27 is shown in Appendix.

Moreover, it can be easily proved that the IULDWGGHM operator also satisfies the properties of monotonicity, idempotency, and boundedness. The proof process can be seen in Appendix.

Theorem 28 (idempotency). If $\widetilde{a}_{i}=\tilde{a}$ for all $i=1, \cdots, n$, then

$$
\begin{aligned}
& \operatorname{IULDWGGHM} M_{w}^{p, q}\left(\tilde{a}_{1}, \cdots, \tilde{a}_{n}\right) \\
& =\operatorname{IULDWGGHM} M_{w}^{p, q}(\widetilde{a}, \cdots, \tilde{a})=\tilde{a}
\end{aligned}
$$

Theorem 29 (monotonicity). Let $\widetilde{\alpha}_{i}=\left[U L_{\alpha_{i}},\left(u_{\alpha_{i}}, v_{\alpha_{i}}\right)\right]$ and $\widetilde{\beta}_{i}=\left[U L_{\beta_{i}},\left(u_{\beta_{i}}, v_{\beta_{i}}\right)\right]$ be any two collections of IULNs, if $u_{\alpha_{i}} \leq$ $u_{\beta_{i}}, v_{\alpha_{i}} \geq v_{\beta_{i}}, U L_{\alpha_{i}} \leq U L_{\beta_{i}}$ for all $i=1, \cdots, n$, then

$$
\begin{aligned}
& \operatorname{IULDWGGHM} M_{w}^{p, q}\left(\widetilde{\alpha}_{1}, \cdots, \widetilde{\alpha}_{n}\right) \\
& \quad \leq \operatorname{IULDWGGHM}{ }_{w}^{p, q}\left(\widetilde{\beta}_{1}, \cdots, \widetilde{\beta}_{n}\right)
\end{aligned}
$$

Theorem 30 (boundedness). The IULDWGHM operator lies between the maximum operator and the minimum operator, i.e.

$$
\begin{aligned}
& \widetilde{\alpha}_{*}=\min \left(\widetilde{\alpha}_{1}, \cdots, \widetilde{\alpha}_{n}\right) \\
& \leq \operatorname{IULDWGGHM} \mathrm{w}_{w}^{p, q}\left(\widetilde{\alpha}_{1}, \cdots, \widetilde{\alpha}_{n}\right) \\
& \leq \max \left(\widetilde{\alpha}_{1}, \cdots, \widetilde{\alpha}_{n}\right)=\widetilde{\alpha}^{*}
\end{aligned}
$$

A group of particular cases can be obtained with respect to the parameters $\mathrm{p}$ and $\mathrm{q}$.

Case 6. If $\mathrm{q}=0$, the IULDWGGHM operator degenerates to IUL generalized weighted mean operator.

$$
\begin{aligned}
& \operatorname{IULDWGGHM} M_{w}^{p, 0}\left(\tilde{a}_{1}, \cdots, \widetilde{a}_{n}\right) \\
& =L H^{-1}\left(\frac{1}{p}\left(\bigotimes_{i=1}^{n} p\left(T_{t_{H}}^{t}\left(L H\left(\tilde{a}_{i}\right)\right)^{n w_{i}}\right)\right)^{1 / n}\right) \\
& =\left(L H^{-1}\left(s_{(1 / p)\left(\prod_{i=1}^{n} p b_{i}^{n w_{i}}\right)}^{1 / n}\right),(1\right. \\
& -\left(1-\prod_{i=1}^{n}\left(1-\left(1-u_{i}^{n w_{i}}\right)^{p}\right)^{1 / n}\right)^{1 / p}, \\
& \left.\left.\left(1-\prod_{i=1}^{n}\left(1-\left(1-\left(1-v_{i}\right)^{n w_{i}}\right)^{p}\right)^{1 / n}\right)^{1 / p}\right)\right)
\end{aligned}
$$

Case 7. If $\mathrm{p}=1, \mathrm{q}=0$, the IULDWGGHM operator degenerates to IUL arithmetic weighted mean operator.

$$
\begin{aligned}
& \text { IULDWGGHM } M_{w}^{1,0}\left(\tilde{a}_{1}, \cdots, \tilde{a}_{n}\right) \\
& =L^{-1}\left(\left(\bigotimes_{i=1}^{n}\left(T_{t_{H}}^{t}\left(L H\left(\widetilde{a}_{i}\right)\right)^{n w_{i}}\right)\right)^{1 / n}\right) \\
& =\left(\operatorname{LH}^{-1}\left(s_{\prod_{i=1}^{n} b_{i}^{w_{i}}}\right),\left(\prod_{i=1}^{n} u_{i}^{w_{i}}, 1-\prod_{i=1}^{n}\left(1-v_{i}\right)^{w_{i}}\right)\right)
\end{aligned}
$$

\section{An Approach to the Generalized Multiple Attributes Group Decision Making (GMAGDM) with the IULDWGHM Operator and the IULDWGGHM Operator}

In this section, the dependent operator and some Heronian mean operators are combined in order to manage green products selection. Furthermore, an intuitionistic unbalanced linguistic GMAGDM approach is proposed.

For a generalized multiple attribute group decision making problem, suppose that $A=\left\{A_{1}, \cdots, A_{m}\right\}$ is a set of all possible alternatives, $E=\left\{e_{1}, \cdots, e_{n}\right\}$ is a set of attributes, $D=\left\{d_{1}, \cdots, d_{t}\right\}$ is a set of decision makers and $E_{k} \subseteq E$ is the corresponding attribute set given by the k-th DM, in which $\bigcup_{k=1}^{t} E_{k}=E, E_{k}(k=1, \cdots, t)$ do not have to be exactly the same. $W=\left\{w_{1}, \cdots, w_{n}\right\}$ is a relative weight vector, where $w_{j}$ denotes the weight of the j-th attribute and $w_{j} \geq$ $0, \sum_{j=1}^{n} w_{j}=1 . \lambda=\left\{\lambda_{1}, \cdots, \lambda_{n}\right\}$ is the weight vector of $\mathrm{DM}_{S}$. For an alternative $x_{i}$, the decision maker $d_{k}$ provides his/her evaluation value $\widetilde{r}_{i j}^{(k)}\left(\widetilde{r}_{i j}^{(k)}=\left[U L_{i j}^{(k)},\left(u_{i j}^{(k)}, v_{i j}^{(k)}\right)\right]\right)$ on the attribute $e_{j}$ in form of intuitionistic unbalanced linguistic information. The algorithm can be described as follows:

Step 1. Input the initial evaluated matrix $\widetilde{R}_{k}=\left(\widetilde{r}_{i j}^{(k)}\right)_{m \times\left|E_{k}\right|}$.

Each decision maker $d_{k}$ assesses every alternative $A_{i}$ with respect to each attribute in the form of the intuitionistic unbalanced linguistic number $\widetilde{r}_{i j}^{(k)}$.

Step 2. Translate the intuitionistic unbalanced linguistic variable to the intuitionistic linguistic number. the Eq.

The intuitionistic linguistic number can be obtained using

$$
\widetilde{s}_{i j}^{(k)}=\left[T_{t_{H}}^{t}\left(L H\left(U L_{i j}\right)\right),\left(u_{i j}^{(k)}, v_{i j}^{(k)}\right)\right]
$$

where the LH is the transformation function, as defined in Definition 10.

Step 3. Calculate the dependent weight vector $w_{i j}^{(k)}$ of $\widetilde{r}_{i j}^{(k)}$.

$$
\begin{aligned}
w_{i j}^{(k)}=\frac{\left(1-d\left(\widetilde{r}_{i j}^{(k)}, \bar{r}_{i}^{k}\right) / \sum_{i=1}^{n} d\left(\widetilde{r}_{i j}^{(k)}, \bar{r}_{i}^{k}\right)\right)}{\sum_{i=1}^{n}\left(1-d\left(\widetilde{r}_{i j}^{(k)}, \bar{r}_{i}^{k}\right) / \sum_{i=1}^{n} d\left(\widetilde{r}_{i j}^{(k)}, \bar{r}_{i}^{k}\right)\right)} \\
\quad i=1, \cdots, m ; j=1, \cdots, n ; k=1, \cdots, t
\end{aligned}
$$

Where $d\left(\widetilde{r}_{i j}^{(k)}, \bar{r}_{i}^{(k)}\right)$ is the distance between $\widetilde{r}_{i j}^{(k)}$ and $\bar{r}_{i}^{(k)}$ as defined in Definition 17.

Step 4. Calculate the comprehensive assessment value $\widetilde{z}_{i}^{(k)}$ and $\widetilde{z}_{i}^{(k) \prime}$ of each alternative from each decision maker 
using the IULDWGHM operator or IULDWGGHM operator.

$$
\begin{aligned}
& \widetilde{z}_{i}^{(k)}=\operatorname{IULDWGH} M_{w}^{p, q}\left(\widetilde{r}_{i 1}^{(k)}, \cdots, \widetilde{r}_{i n}^{(k)}\right) \\
& i=1, \cdots, m ; k=1, \cdots, t ; \\
& \widetilde{z}_{i}^{(k) \prime}=\operatorname{IULDWGGH} M_{w}^{p, q}\left(\widetilde{r}_{i 1}^{(k)}, \cdots, \widetilde{r}_{i n}^{(k)}\right) \\
& \quad i=1, \cdots, m ; k=1, \cdots, t ;
\end{aligned}
$$

Step 5. Repeat the Step 3 to get the weight vector $\lambda_{i}^{(k)}$ of the $\mathrm{k}$-th decision maker, which can be counted as

$$
\begin{array}{r}
\lambda_{i}^{(k)}=\frac{\left(1-d\left(\widetilde{z}_{i}^{(k)}, \bar{z}^{(k)}\right) / \sum_{i=1}^{n} d\left(\widetilde{z}_{i}^{(k)}, \bar{z}^{(k)}\right)\right)}{\sum_{i=1}^{n}\left(1-d\left(\widetilde{z}_{i}^{(k)}, \bar{z}^{(k)}\right) / \sum_{i=1}^{n} d\left(\widetilde{z}_{i}^{(k)}, \bar{z}^{(k)}\right)\right)} \\
i=1, \cdots, m ; k=1, \cdots, t
\end{array}
$$

Step 6. Aggregate the final decision values of each alternative using the IULDWGHM operator or IULDWGGHM operator

$$
\widetilde{z}_{i}=\operatorname{IULDWGHM}\left(\widetilde{z}_{i}^{(1)}, \cdots, \widetilde{z}_{i}^{(t)}\right) \quad i=1, \cdots, n
$$

or

$$
\widetilde{z}_{i}^{\prime}=\operatorname{IULDWGGHM}\left(\widetilde{z}_{i}^{(1) \prime}, \cdots, \widetilde{z}_{i}^{(t) \prime}\right) \quad i=1, \cdots, n .
$$

Step 7. Rank all alternatives $x_{i}(i=1, \cdots, n)$ according to corresponding score values and accuracy values on the basis of Eq. (21), (22).

\section{A Case Study for the Green Products Selection Problem}

In this section, the proposed intuitionistic unbalanced linguistic GMAGDM model is applied to the green products selection problem.

With the continuing development of the society, many companies have been developed to reduce environment pollution of waste materials. Furthermore, the public have realized the importance of the green products for environment protection. In order to find a balance between the public satisfaction and the environment protection, it is important for companies to select the best green product.

A decision maker would like to have an investment in green product companies, four green product companies will be considered which are the green food company $\left(A_{1}\right)$, the green packing company $\left(A_{2}\right)$, the green bicycle company $\left(A_{3}\right)$, the green building material company $\left(A_{4}\right)$. Three expert are composed of the resource expert, the environmental policy expert and the management expert which can be denoted fvas $\mathrm{D}=\left\{\mathrm{d}_{1}, \mathrm{~d}_{2}, \mathrm{~d}_{3}\right\}$. They use five attributes to assess the alternatives: material factor $\mathrm{e}_{1}$, re-usability factor $e_{2}$, contamination factor $e_{3}$, environment pollution factor $e_{4}$ and public satisfaction factor $\mathrm{e}_{5}$.

The corresponding attribute sets of three experts are $A_{1}=\left\{e_{1}, e_{2}, e_{3}\right\}, A_{2}=\left\{e_{1}, e_{2}, e_{3}, e_{4}\right\}, A_{3}=\left\{e_{1}, e_{2}, e_{3}, e_{4}, e_{5}\right\}$.
TABLE 2: The intuitionistic unbalanced linguistic decision matrix provided by $d_{1}$.

\begin{tabular}{cccc}
\hline & $e_{1}$ & $e_{2}$ & $e_{3}$ \\
\hline$A_{1}$ & {$[A H,(0.2,0.6)]$} & {$[M,(0.4,0.5)]$} & {$[M,(0.2,0.7)]$} \\
$A_{2}$ & {$[M,(0.3,0.7)]$} & {$[L,(0.1,0.8)]$} & {$[L,(0.1,0.8)]$} \\
$A_{3}$ & {$[M,(0.2,0.7)]$} & {$[L,(0.1,0.8)]$} & {$[M,(0.2,0.7)]$} \\
$A_{4}$ & {$[M,(0.2,0.7)]$} & {$[M,(0.3,0.6)]$} & {$[A H,(0.4,0.5)]$} \\
\hline
\end{tabular}

Finally, the four green projects are to be assessed using the unbalanced linguistic term set $S=\{N$ (none), $L$ (low), $M$ (medium), AH (almost high), $H$ (high), VH (very high), $T$ (total)\}. Supposing that

$$
\begin{aligned}
L H & =l(1,3) \bigcup l(2,5) \bigcup l(3,9)=\left\{s_{0}^{3}, s_{1}^{3}, s_{2}^{3}\right\} \\
\cdot & \bigcup\left\{s_{0}^{5}, s_{1}^{5}, s_{2}^{5}, s_{3}^{5}, s_{4}^{5}\right\} \\
\cdot & \bigcup\left\{s_{0}^{9}, s_{1}^{9}, s_{2}^{9}, s_{3}^{9}, s_{4}^{9}, s_{5}^{9}, s_{6}^{9}, s_{7}^{9}, s_{8}^{9}\right\}, \\
t_{H} & =3
\end{aligned}
$$

The assessments are listed in Tables 2, 3, and 4, denoted as $\widetilde{R}_{1}=\left(\widetilde{r}_{i j}^{(1)}\right)_{4 \times 3}, \widetilde{R}_{2}=\left(\widetilde{r}_{i j}^{(2)}\right)_{4 \times 4}, \widetilde{R}_{3}=\left(\widetilde{r}_{i j}^{(3)}\right)_{4 \times 5}$ which are represented by IULNs.

Based on the linguistic hierarchies, the semantic representation of the unbalanced linguistic variables are gained as follows.

$$
\begin{array}{r}
N=s_{0}^{5}, \\
L=s_{1}^{5}, \\
M=s_{2}^{5}, \\
A H=s_{5}^{9}, \\
H=s_{6}^{9}, \\
Q H=s_{7}^{9}, \\
T=s_{8}^{9} .
\end{array}
$$

Utilize dependent operator (64), the weight vector of attributes are obtained as

$$
\begin{aligned}
& w_{1}=\left(\begin{array}{lll}
0.4823 & 0.3014 & 0.2163 \\
0.2125 & 03937 & 0.3937 \\
0.3597 & 0.2807 & 0.3597 \\
0.3059 & 0.4499 & 0.2442
\end{array}\right), \\
& w_{2}=\left(\begin{array}{llll}
0.2734 & 0.2985 & 0.2734 & 0.1548 \\
0.1675 & 0.3008 & 0.3241 & 0.2075 \\
0.2714 & 0.2854 & 0.1718 & 0.2714 \\
0.2778 & 0.1846 & 0.3136 & 0.2240
\end{array}\right),
\end{aligned}
$$


TABle 3: The intuitionistic unbalanced linguistic decision matrix provided by $d_{2}$.

\begin{tabular}{lcccc}
\hline & $e_{1}$ & $e_{2}$ & $e_{3}$ & $e_{4}$ \\
\hline$A_{1}$ & {$[M,(0.1,0.7)]$} & {$[M,(0.2,0.7)]$} & {$[M,(0.2,0.8)]$} & {$[H,(0.4,0.5)]$} \\
$A_{2}$ & {$[A H,(0.4,0.5)]$} & {$[M,(0.3,0.6)]$} & {$[A H,(0.2,0.6)]$} & {$[M,(0.2,0.7)]$} \\
$A_{3}$ & {$[M,(0.2,0.6)]$} & {$[A H,(0.2,0.7)]$} & {$[A H,(0.4,0.6)]$} & {$[M,(0.3,0.7)]$} \\
$A_{4}$ & {$[A H,(0.3,0.6)]$} & {$[H,(0.4,0.5)]$} & {$[H,(0.3,0.6)]$} & {$[A H,(0.2,0.6)]$} \\
\hline
\end{tabular}

TABLE 4: The intuitionistic unbalanced linguistic decision matrix provided by $d_{3}$.

\begin{tabular}{cccccc}
\hline & $e_{1}$ & $e_{2}$ & $e_{3}$ & $e_{4}$ & $e_{5}$ \\
\hline$A_{1}$ & {$[A H,(0.2,0.7)]$} & {$[L,(0.4,0.6)]$} & {$[A H,(0.5,0.5)]$} & {$[M,(0.2,0.6)]$} & {$[M,(0.3,0.4)]$} \\
$A_{2}$ & {$[M,(0.4,0.6)]$} & {$[A H,(0.4,0.5)]$} & {$[M,(0.1,0.8)]$} & {$[M,(0.5,0.5)]$} & {$[A H,(0.3,0.6)]$} \\
$A_{3}$ & {$[M,(0.2,0.7)]$} & {$[M,(0.2,0.7)]$} & {$[A H,(0.3,0.7)]$} & {$[A H,(0.2,0.7)]$} & {$[A H,(0.3,0.4)]$} \\
$A_{4}$ & {$[V H,(0.5,0.4)]$} & {$[M,(0.2,0.8)]$} & {$[H,(0.3,0.6)]$} & {$[A H,(0.3,0.6)]$} & {$[A H,(0.5,0.3)]$} \\
\hline
\end{tabular}

$$
w_{3}=\left(\begin{array}{lllll}
0.2202 & 0.1771 & 0.1601 & 0.2154 & 0.2271 \\
0.2410 & 0.1825 & 0.1232 & 0.2119 & 0.2414 \\
0.1957 & 0.1957 & 0.2391 & 0.2283 & 0.1412 \\
0.1658 & 0.1657 & 0.2375 & 0.2182 & 0.2128
\end{array}\right)
$$

Let $p=q=0.5$, then the comprehensive assessment values of each alternative by IULDWGHM operator are as follows

$$
\begin{aligned}
z_{1}^{1} & =\left[L H^{-1}\left(s_{4.3265}\right),(0.2569,0.6018)\right], \\
z_{2}^{1} & =\left[L H^{-1}\left(s_{2.4244}\right),(0.1444,0.7778)\right], \\
z_{3}^{1} & =\left[L H^{-1}\left(s_{3.3373}\right),(0.1676,0.7316)\right], \\
z_{4}^{1} & =\left[L H^{-1}\left(s_{4.2163}\right),(0.2916,0.6079)\right], \\
z_{1}^{2} & =\left[L H^{-1}\left(s_{4.3027}\right),(0.2049,0.6920)\right], \\
z_{2}^{2} & =\left[L H^{-1}\left(s_{4.4201}\right),(0.2632,0.6075)\right], \\
z_{3}^{2} & =\left[L H^{-1}\left(s_{4.4283}\right),(0.2636,0.6259)\right], \\
z_{4}^{2} & =\left[L H^{-1}\left(s_{5.4446}\right),(0.2943,0.5814)\right], \\
z_{1}^{3} & =\left[L H^{-1}\left(s_{3.9280}\right),(0.3091,0.5617)\right], \\
z_{2}^{3} & =\left[L H^{-1}\left(s_{4.3541}\right),(0.3567,0.5362)\right], \\
z_{3}^{3} & =\left[L H^{-1}\left(s_{4.5627}\right),(0.2361,0.6510)\right], \\
z_{4}^{3} & =\left[L H^{-1}\left(s_{5.3402}\right),(0.3567,0.5362)\right] .
\end{aligned}
$$

Let $p=q=0.5$, then the collective assessment values of each alternative by IULDWGGHM operator are as follows

$$
\begin{aligned}
& z_{1}^{1 \prime}=\left[L H^{-1}\left(s_{4.7535}\right),(0.4615,0.4629)\right], \\
& z_{2}^{1 \prime}=\left[L H^{-1}\left(s_{2.3178}\right),(0.3709,0.5901)\right],
\end{aligned}
$$

$$
\begin{aligned}
z_{3}^{1 \prime} & =\left[L H^{-1}\left(s_{3.4050}\right),(0.3722,0.5583)\right], \\
z_{4}^{1 \prime} & =\left[L H^{-1}\left(s_{4.2978}\right),(0.4281,0.5352)\right], \\
z_{1}^{2 \prime} & =\left[L H^{-1}\left(s_{4.2944}\right),(0.3723,0.5361)\right], \\
z_{2}^{2 \prime} & =\left[L H^{-1}\left(s_{4.6118}\right),(0.4654,0.4969)\right], \\
z_{3}^{2 \prime} & =\left[L H^{-1}\left(s_{4.4886}\right),(04176,0.5072)\right], \\
z_{4}^{2 \prime} & =\left[L H^{-1}\left(s_{5.6152}\right),(0.4762,0.5007)\right], \\
z_{1}^{3 \prime} & =\left[L H^{-1}\left(s_{3.9763}\right),(0.4551,0.5567)\right], \\
z_{2}^{3 \prime} & =\left[L H^{-1}\left(s_{4.5127}\right),(0.4741,0.5174)\right], \\
z_{3}^{3 \prime} & =\left[L H^{-1}\left(s_{4.6699}\right),(0.4177,0.5533)\right], \\
z_{2}^{3 \prime} & =\left[L H^{-1}\left(s_{4.5127}\right),(0.4741,0.5174)\right] .
\end{aligned}
$$

By the Eq. (67), we could have the weight vector of decision makers, denote as $\lambda_{i}^{(k)}$ and $\lambda_{i}^{(k) \prime}$ of $z_{i}^{(k)}$ and $z_{i}^{(k) \prime}$.

$$
\begin{gathered}
\lambda_{i}^{(k)}=\left(\begin{array}{lll}
0.4162 & 0.2257 & 0.3581 \\
0.2773 & 0.3910 & 0.3317 \\
0.2646 & 0.3645 & 0.3709 \\
0.2556 & 0.4467 & 0.2977
\end{array}\right), \\
\lambda_{i}^{(k) \prime}=\left(\begin{array}{lll}
0.2492 & 0.3784 & 0.3724 \\
0.2666 & 0.3561 & 0.3772 \\
0.2561 & 0.3848 & 0.3791 \\
0.2542 & 0.2888 & 0.4570
\end{array}\right) .
\end{gathered}
$$

The final assessment values of each alternative by IULDWGHM operator and IULDWGGHM operator are shown in Table 5. 
TABLE 5: The collective assessment values by IULDWGHM and IULDWGGHM operator.

\begin{tabular}{lc}
\hline IULDWGHM & IULDWGGHM \\
\hline$\widetilde{z}_{1}=\left[L H^{-1}\left(s_{4.1305}\right),(0.2578,0.6164)\right]$ & $\widetilde{z}_{1}^{\prime}=\left[L H^{-1}\left(s_{4.3150}\right),(0.4725,0.5080)\right]$ \\
$\widetilde{z}_{2}=\left[L H^{-1}\left(s_{2.4244}\right),(0.1444,0.7778)\right]$ & $\widetilde{z}_{2}^{\prime}=\left[L H^{-1}\left(s_{3.9626}\right),(0.4574,0.5348)\right]$ \\
$\widetilde{z}_{3}=\left[L H^{-1}\left(s_{4.1293}\right),(0.2241,0.6779)\right]$ & $\widetilde{z}_{3}^{\prime}=\left[L H^{-1}\left(s_{4.3469}\right),(0.4609,0.5253)\right]$ \\
$\widetilde{z}_{4}=\left[L H^{-1}\left(s_{4.9969}\right),(0.3098,0.5792)\right]$ & $\widetilde{z}_{4}^{\prime}=\left[L H^{-1}\left(s_{5.4063}\right),(0.4571,0.5332)\right]$ \\
\hline
\end{tabular}

TABLE 6: The ordering of alternatives by the IULDWGHM operator and the IULDWGGHM operator.

\begin{tabular}{llr}
\hline$p$ and $q$ & \multicolumn{1}{c}{ IULDWGHM } & \multicolumn{1}{c}{ IULDWGGHM } \\
\hline$p=1, q=1$ & $A_{4}>A_{1}>A_{3}>A_{2}$ & $A_{4}>A_{1}>A_{3}>A_{2}$ \\
$p=0, q=1$ & $A_{4}>A_{2}>A_{3}>A_{1}$ & $A_{4}>A_{2}>A_{3}>A_{1}$ \\
$p=0.2, q=1.8$ & $A_{4}>A_{2}>A_{3}>A_{1}$ & $A_{4}>A_{2}>A_{3}>A_{1}$ \\
$p=0.4, q=1.6$ & $A_{4}>A_{3}>A_{2}>A_{1}$ & $A_{4}>A_{3}>A_{2}>A_{1}$ \\
$p=0.6, q=1.4$ & $A_{4}>A_{3}>A_{1}>A_{2}$ & $A_{4}>A_{3}>A_{1}>A_{2}$ \\
$p=0.8, q=1.2$ & $A_{4}>A_{3}>A_{1}>A_{2}$ & $A_{4}>A_{3}>A_{1}>A_{2}$ \\
$p=1.2, q=0.8$ & $A_{4}>A_{1}>A_{3}>A_{2}$ & $A_{4}>A_{1}>A_{3}>A_{2}$ \\
$p=1, q=0$ & $A_{4}>A_{1}>A_{3}>A_{2}$ & $A_{4}>A_{1}>A_{3}>A_{2}$ \\
\hline
\end{tabular}

The score values by IULDWGHM operator and IULDWGGHM operator are

$$
\begin{aligned}
& S\left(\widetilde{z}_{1}\right)=0.1656 \\
& S\left(\widetilde{z}_{2}\right)=0.1391, \\
& S\left(\widetilde{z}_{3}\right)=0.1410 \\
& S\left(\widetilde{z}_{4}\right)=0.2282 \\
& S\left(\widetilde{z}_{1}^{\prime}\right)=0.2601, \\
& S\left(\widetilde{z}_{2}^{\prime}\right)=0.2285 \\
& S\left(\widetilde{z}_{3}^{\prime}\right)=0.2542 \\
& S\left(\widetilde{z}_{4}^{\prime}\right)=0.3122 .
\end{aligned}
$$

From above result, we could have the ranking of the alternatives by IULDWGGHM operator

$$
S\left(\widetilde{z}_{4}\right)>S\left(\widetilde{z}_{1}\right)>S\left(\widetilde{z}_{3}\right)>S\left(\widetilde{z}_{2}\right) ;
$$

Similarly, the ranking of the alternatives by IULDWGGHM operator is

$$
S\left(\widetilde{z}_{4}^{\prime}\right)>S\left(\widetilde{z}_{1}^{\prime}\right)>S\left(\widetilde{z}_{3}^{\prime}\right)>S\left(\widetilde{z}_{2}^{\prime}\right)
$$

From above analysis, the ranking of alternatives by the IULDWGHM operator is that $A_{4}>A_{1}>A_{3}>A_{2}$ and the ranking of alternatives by the IULDWGGHM operator is that $A_{4}>A_{1}>A_{3}>A_{2}$. Thus the best green product project is $\mathrm{A}_{4}$ based on two methods. In order to illustrate the impact of the parameters $\mathrm{p}$ and $\mathrm{q}$ on aggregation results, we take the different values of $\mathrm{p}$ and $\mathrm{q}$, the rankings of alternatives based on the IULDWGHM operator and the IULDWGGHM operator can be obtained, which are shown in Table 6 .
It is worth noted that most of the values obtained by the IULDWGHM operator are smaller than the values obtained by the IULDWGGHM operator, which indicates that the IULDWGHM operator can obtain more unfavorable (or pessimistic) expectations, while the IULDWGGHM operator has more favorable (or optimistic) expectations. Therefore, we can conclude that the IULDWGHM operator can be considered as the pessimistic one, while the IULDWGGHM operator can be considered as the optimistic one and the values of the parameters can be considered as the pessimistic or optimistic levels. So, we can conclude that the decision makers who take a gloomy view of the prospects could use the IULDWGHM operator and choose the smaller values of the parameter $\mathrm{p}$ and $\mathrm{q}$, while the decision makers who are optimistic could use the IULDWGGHM operator and choose the bigger values of the parameter $\mathrm{p}$ and $\mathrm{q}$.

\section{Comparison Analyses and Discussion}

To demonstrate the feasibility and applicability of the proposed intuitionistic unbalanced linguistic GMAGDM method, a series of comparative studies have been implemented with the relevant common aggregation method and classical decision making approach.

6.1. Comparison with the Existing Linguistic Aggregation Operators. We will compare our methods with previous linguistic aggregation operators such as the dependent intuitionistic linguistic generalized ordered weighted average (DILGOWA) operator and the dependent intuitionistic linguistic generalized ordered weight geometric (DILGOWG) operator, the intuitionistic linguistic dependent weighted generalized Bonferroni mean (ILDWGBM) operator and the intuitionistic linguistic dependent weighted generalized geometric Bonferroni mean (ILDWGGBM) operator.

To select the optimal alternative with the DILGOWA, DILGOWG, ILDWGBM and the ILDWGGBM operators, we 
TABLE 7: Comparison with the exiting linguistic operators.

\begin{tabular}{lccc}
\hline Aggregation operators & Linguistic distribution & Parameter number & Order of alternative \\
\hline DILGOWA operator & Balanced & One & $A_{4}>A_{1}>A_{3}>A_{2}$ \\
DILGOWG operator & Balanced & One & $A_{4}>A_{1}>A_{3}>A_{2}$ \\
ILDWGBM operator & Balanced & Two & $A_{4}>A_{3}>A_{1}>A_{2}$ \\
ILDWGGBM operator & Balanced & Two & $A_{4}>A_{3}>A_{1}>A_{2}$ \\
IULDWGHM operator & Unbalanced & Two & $A_{4}>A_{1}>A_{3}>A_{2}$ \\
IULDWGGHM operator & Unbalanced & Two & $A_{4}>A_{1}>A_{3}>A_{2}$ \\
\hline
\end{tabular}

should transform the evaluation values into 2-tuple linguistic information which are as follows:

$$
\begin{gathered}
N \longrightarrow\left(s_{0}^{5}, 0\right), \\
L \longrightarrow\left(s_{1}^{5}, 0\right), \\
M \longrightarrow\left(s_{2}^{5}, 0\right), \\
A H \longrightarrow\left(s_{5}^{9}, 0\right), \\
H \longrightarrow\left(s_{6}^{9}, 0\right), \\
Q H \longrightarrow\left(s_{7}^{9}, 0\right), \\
T \longrightarrow\left(s_{8}^{9}, 0\right) ;
\end{gathered}
$$

After calculating the dependent weights $w_{11}^{(1)}=0.4823$, $w_{12}^{(1)}=0.3014, w_{13}^{(1)}=0.2163, w_{21}^{(1)}=0.2125, w_{22}^{(1)}=0.3937$, $w_{23}^{(1)}=0.3937, w_{31}^{(1)}=0.3597, w_{32}^{(1)}=0.2807, w_{33}^{(1)}=0.3597$, $w_{41}^{(1)}=0.3059, w_{42}^{(1)}=0.4499, w_{43}^{(1)}=0.2442, w_{11}^{(2)}=0.2734$, $w_{12}^{(2)}=0.2985, w_{13}^{(2)}=0.2734, w_{14}^{(2)}=0.1548, w_{21}^{(2)}=0.1675$, $w_{22}^{(2)}=0.3008, w_{23}^{(2)}=0.3241, w_{24}^{(2)}=0.2075, w_{31}^{(2)}=0.2714$, $w_{32}^{(2)}=0.2854, w_{33}^{(2)}=0.1718, w_{34}^{(2)}=0.2714, w_{41}^{(2)}=0.2778$, $w_{42}^{(2)}=0.1846, w_{43}^{(2)}=0.3136, w_{44}^{(2)}=0.2240, w_{11}^{(3)}=0.2202$, $w_{12}^{(3)}=0.1771, w_{13}^{(3)}=0.1601, w_{14}^{(3)}=0.2154, w_{15}^{(3)}=0.2271$, $w_{21}^{(3)}=0.2410, w_{22}^{(3)}=0.1825, w_{23}^{(3)}=0.1232, w_{24}^{(3)}=0.2119$, $w_{25}^{(3)}=0.2414, w_{31}^{(3)}=0.1956, w_{32}^{(3)}=0.1957, w_{33}^{(3)}=0.2391$, $w_{34}^{(3)}=0.2283, w_{35}^{(3)}=0.1412, w_{41}^{(3)}=0.1658, w_{42}^{(3)}=0.1657$, $w_{43}^{(3)}=0.2375, w_{44}^{(3)}=0.2182, w_{45}^{(3)}=0.2128$, the overall collective preference can be obtained. The final results are shown in Table $7(\lambda=1, p=q=1)$.

From the results of different aggregation operators, we ca see that the optimal alternative is the same one, i.e., the best alternative is $A_{4}$ which indicates the validity and rationality of our proposed method. Furthermore, our proposed approach considers the interactions between not only criteria value $e_{i}$ and $e_{j}(i<j)$ but also between $e_{i}$ and itself and has flexible parameters $\mathrm{p}$ and $\mathrm{q}$ which can satisfies the real demand effectively.

6.2. The TOPSIS Method for Intuitionistic Unbalanced Linguistic GMAGMD. In this section, we will extend the classical TOPSIS method to the intuitionistic unbalanced linguistic environment. The basic principle of the TOPSIS method is that the optimal alternative should have the farthest distance from the negative ideal solution and the closest distance from the positive ideal solution simultaneously. The steps are involved by using the TOPSIS method.

Step 8. Obtain the 2-tuple linguistic representation of unbalanced linguistic terms shown in Section 6.1;

Step 9. Definite the intuitionistic unbalanced linguistic positive ideal solution (IULPIS) and the intuitionistic unbalanced linguistic negative ideal solution (IULNIS) according to Definition 16. Since the unbalanced linguistic term set is $S=\{N$ (none), $L$ (low), $M$ (medium), AH (almost high), $H$ (high), $\mathrm{VH}$ (very high), $\mathrm{T}$ (total)\}, based on the comprehensive assessment of IULDWGHM operator and Definition 16, Thus the IULPIS IUd IULNIS $_{S}$ of $d_{1}, d_{2}, d_{3}$ are

$$
\begin{aligned}
& r^{(1)+}=\left[L H^{-1}\left(s_{4.2163}\right),(0.2916,0.6079)\right], \\
& r^{(1)-}=\left[L H^{-1}\left(s_{4.2163}\right),(0.2916,0.6079)\right], \\
& r^{(2)+}=\left[L H^{-1}\left(s_{5.4446}\right),(0.2943,0.5814)\right], \\
& r^{(2)-}=\left[L H^{-1}\left(s_{4.3027}\right),(0.2049,0.6920)\right], \\
& r^{(3)+}=\left[L H^{-1}\left(s_{5.3402}\right),(0.3567,0.5362)\right], \\
& r^{(3)-}=\left[L H^{-1}\left(s_{4.5627}\right),(0.2361,0.6510)\right] .
\end{aligned}
$$

Step 10. Calculate the distance from each evaluation value to IULPIS $_{s}$ and IULNIS $s$ using the following equation

$$
\begin{aligned}
& d_{i}^{+}=\sum_{j=1}^{n} w_{j} d\left(r_{i j}, r^{(j)+}\right), \\
& d_{i}^{-}=\sum_{j=1}^{n} w_{j} d\left(r_{i j}, r^{(j)-}\right)
\end{aligned}
$$

Where the separation between alternatives is the Hamming distance, i.e. $d\left(\widetilde{a}_{i}, \widetilde{a}_{j}\right)=\left(1 /\left(2 G\left(t_{H}\right)-1\right)\right) \mid\left(1+u_{i}-\right.$ $\left.\left.v_{i}\right) \Delta^{-1}\left(T_{t_{H}}^{t}\left(L H\left(U L_{i}\right)\right)\right)-\left(1+u_{j}-v_{j}\right) \Delta^{-1}\left(T_{t_{H}}^{t}\left(L H\left(U L_{j}\right)\right)\right)\right)$, and the weight vector of decision makers is $W=$ $(0.5376,0.1809,0.2815)^{T}$, then we can get $d_{i}^{+}$and $d_{i}^{-}$. Obviously, the larger $d_{i}^{-}$and the smaller $d_{i}^{+}$are, the better the alternative is.

Step 11. Calculate the closeness coefficient to ideal solution as

$$
C C_{i}=\frac{d_{i}^{-}}{d_{i}^{+}+d_{i}^{-}}, \quad i=1,2,3,4
$$


The $C C_{i}$ for the alternative $A_{i}$ can be got as

$$
\begin{aligned}
& C C_{1}=\frac{d_{1}^{-}}{d_{1}^{+}+d_{1}^{-}}=0.1408 \\
& C C_{2}=\frac{d_{2}^{-}}{d_{2}^{+}+d_{2}^{-}}=0.0553, \\
& C C_{3}=\frac{d_{3}^{-}}{d_{3}^{+}+d_{3}^{-}}=0.0675, \\
& C C_{4}=\frac{d_{4}^{-}}{d_{4}^{+}+d_{4}^{-}}=1 .
\end{aligned}
$$

According to the closeness coefficient, we can determine the ranking of all alternatives as $A_{4}>A_{1}>A_{3}>A_{2}$, the best alternative is $A_{4}$.

Obviously, the ranking of alternatives obtained by the intuitionistic unbalanced linguistic TOPSIS method is identical to that by the IULDWGHM and IULDWGGHM aggregation operators, which states the validity of the proposed method in this paper. Thus, response solution $A_{4}$ is the most appropriate one.

It is clear that all comparison approaches based on frequently-used aggregated operators and TOPSIS method can lead to the identical result with GMAGDM method proposed in this paper. The discussions are as follows:

(1) The evaluation information of this paper is in form of intuitionistic unbalanced linguistic terms, which can describe the indeterminate and incomplete assessments more effectively.

(2) The aggregated method in this paper not only reflects the correlation of all attributes, but also has flexible parameters to satisfy the fuzzy complex decision making environment. Thus, the proposed method is flexible;

(3) We develop a model to deal with the situation where the weights information is unknown. The proposed model for dependent weight vector is advantaged and effective, which relieves the influence of unfair evaluations and takes objective weights information into consideration.
In summary, the proposed method would be more comfortable to handle uncertain information and intuitionistic unbalanced information in complex decision-making problems. Therefore, it is more reasonable than other existing methods.

\section{Conclusions}

This paper focuses on GMAGDM problem with intuitionistic unbalanced linguistic information, which is more conformable to the practical situation. Considering the correlation of input arguments and the impact of unfairness by the personal preferences of decision makers, we have introduced some new dependent weighted Heronian mean aggregation functions in intuitionistic unbalanced linguistic environment. The intuitionistic unbalanced linguistic dependent operator has been proposed to establish the weight vectors. Then the IULDWGHM operator and the IULGDWGGHM operator have been developed to aggregate the individual preference to a collective preference. An algorithm for intuitionistic unbalanced linguistic generalized multiple attribute group decision making with completely unknown weight information are developed subsequently. Some main properties and particular cases of the operators have been studied. A green product selection case is given to illustrate the effectiveness and universality of the developed approach.

In the future, we expect to extend the IULDWGHM operator and the IULDWGGHM operator to more complicated situations, such as interval linguistic information, hesitant fuzzy linguistic environment, probabilistic fuzzy linguistic information and consider other applications.

\section{Appendix}

\section{A. The Proof of Lemma 26}

Proof. we prove Eq. (55) by means of mathematical induction. For $n=2$, according to Eqs. (2) - (5), we have

$$
\begin{aligned}
& \bigotimes_{i=1}^{2}\left(\left(p\left(T_{t_{H}}^{t}\left(L H\left(\tilde{a}_{i}\right)\right)\right)\right)^{2 w_{i}} \oplus\left(q\left(T_{t_{H}}^{t}\left(L H\left(\widetilde{a}_{2}\right)\right)\right)\right)^{2 w_{j}}\right) \\
& \left.\quad=\left[s_{\prod_{i=1}^{2}\left(p b_{i}^{2 w_{i}}+q b_{2}^{2 w_{2}}\right)}\right)\left(\prod_{i=1}^{2}\left(1-\left(1-u_{i}^{2 w_{i}}\right)^{p}\left(1-u_{2}^{2 w_{2}}\right)^{q}, 1-\prod_{i=1}^{2}\left(1-\left(1-\left(1-v_{i}\right)^{2 w_{i}}\right)^{p}\left(1-\left(1-v_{2}\right)^{2 w_{2}}\right)^{q}\right)\right)\right)\right] .
\end{aligned}
$$

Therefore, Eq. (55) holds for $n=2$.
If Eq. (55) holds for $n=k$,

Then when $n=k+1$, we can obtain

$$
\begin{gathered}
\bigotimes_{i=1}^{k+1}\left(\left(p\left(T_{t_{H}}^{t}\left(L H\left(\tilde{a}_{i}\right)\right)\right)^{(k+1) w_{i}}\right) \oplus\left(q\left(T_{t_{H}}^{t}\left(L H\left(\widetilde{a}_{k+1}\right)\right)\right)\right)^{(k+1) w_{k+1}}\right) \\
=\bigotimes_{i=1}^{k}\left(\left(p\left(T_{t_{H}}^{t}\left(L H\left(\widetilde{a}_{i}\right)\right)\right)^{k w_{i}}\right) \oplus\left(q\left(T_{t_{H}}^{t}\left(L H\left(\widetilde{a}_{k}\right)\right)\right)\right)^{k w_{k}}\right)
\end{gathered}
$$


Mathematical Problems in Engineering

19

$$
\begin{aligned}
& \otimes\left(\left(p\left(T_{t_{H}}^{t}\left(L H\left(\widetilde{a}_{i}\right)\right)\right)^{(k+1) w_{i}}\right) \oplus\left(q\left(T_{t_{H}}^{t}\left(L H\left(\widetilde{a}_{k+1}\right)\right)\right)\right)^{(k+1) w_{k+1}}\right)
\end{aligned}
$$

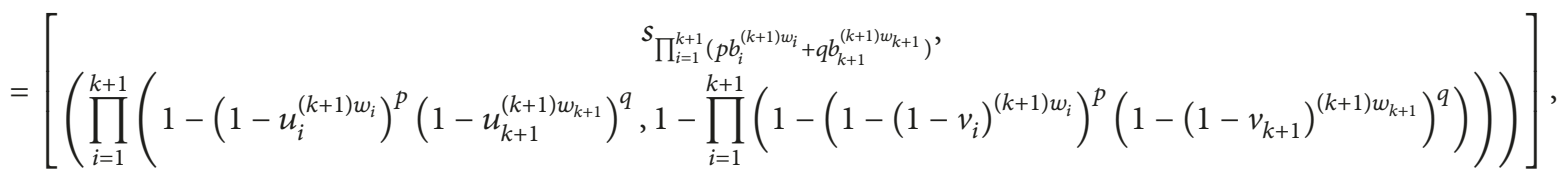

(A.2)

which means that Eq. (55) also holds for $n=k+1$. We complete the proof.

B. The Proof of Theorem 27

Proof. by Eq. (4) (6), we can have

$$
\begin{aligned}
& p\left(T_{t_{H}}^{t}\left(L H\left(\widetilde{a}_{i}\right)\right)\right)^{n w_{i}}=\left[s_{p b_{i}^{n w_{i}}, 1-\left(1-u_{i}^{n w_{i}}\right)^{p},(1}\right. \\
& \left.\left.\quad-\left(1-v_{i}\right)^{n w_{i}}\right)^{p}\right] ; \\
& q\left(T_{t_{H}}^{t}\left(L H\left(\widetilde{a}_{j}\right)\right)\right)^{n w_{j}}=\left[s_{q b_{j} w_{j}, 1-\left(1-u_{j}^{n w_{j}}\right)^{q},(1}\right. \\
& \left.\left.\quad-\left(1-v_{j}\right)^{n w_{j}}\right)^{q}\right] ; \\
& p\left(T_{t_{H}}^{t}\left(L H\left(\widetilde{a}_{i}\right)\right)\right)^{n w_{i}} \oplus q\left(T_{t_{H}}^{t}\left(L H\left(\widetilde{a}_{j}\right)\right)\right)^{n w_{j}} \\
& \quad=\left[s_{p b_{i}^{n w_{i}}+q b_{j}}^{n w_{j},},\left(1-\left(1-u_{i}^{n w_{i}}\right)^{p}\right.\right. \\
& \left.\left.\quad \cdot\left(1-u_{j}^{n w_{j}}\right)^{q},\left(1-\left(1-v_{i}\right)^{n w_{i}}\right)^{p}\left(1-\left(1-v_{j}\right)^{n w_{j}}\right)^{q}\right)\right]
\end{aligned}
$$

(B.1)

In the following, we need to prove following Eq. (B.2) by mathematical induction on $\mathrm{n}$.

$$
\begin{aligned}
& \bigotimes_{i=1}^{n} \bigotimes_{j=i}^{n}\left(p\left(T_{t_{H}}^{t}\left(L H\left(\widetilde{a}_{i}\right)\right)\right)^{n w_{i}} \oplus q\left(T_{t_{H}}^{t}\left(L H\left(\widetilde{a}_{j}\right)\right)\right)^{n w_{j}}\right) \\
& =\left[s_{\prod_{i=1}^{n} \prod_{j=i}^{n}\left(p b_{i}^{n w_{i}}+q b_{j}^{n w_{j}}\right)},\right. \\
& \left(\prod_{i=1}^{n} \prod_{j=i}^{n}\left(1-\left(1-u_{i}^{n w_{i}}\right)^{p}\left(1-u_{j}^{n w_{j}}\right)^{q}\right), 1\right. \\
& \left.\left.-\prod_{i=1}^{n} \prod_{j=i}^{n}\left(1-\left(1-\left(1-v_{i}\right)^{n w_{i}}\right)^{p}\left(1-\left(1-v_{j}\right)^{n w_{j}}\right)^{q}\right)\right)\right]
\end{aligned}
$$

For $\mathrm{n}=2$,

$$
\begin{gathered}
\bigotimes_{i=1}^{2} \bigotimes_{j=i}^{2}\left(p\left(T_{t_{H}}^{t}\left(L H\left(\widetilde{a}_{i}\right)\right)\right)^{2 w_{i}}\right. \\
\left.\oplus q\left(T_{t_{H}}^{t}\left(L H\left(\widetilde{a}_{j}\right)\right)\right)^{2 w_{j}}\right)
\end{gathered}
$$

$$
\begin{aligned}
& =\left(p\left(T_{t_{H}}^{t}\left(L H\left(\widetilde{a}_{1}\right)\right)\right)^{2 w_{1}} \oplus q\left(T_{t_{H}}^{t}\left(L H\left(\widetilde{a}_{1}\right)\right)\right)^{2 w_{1}}\right) \\
& \otimes\left(p\left(T_{t_{H}}^{t}\left(L H\left(\widetilde{a}_{1}\right)\right)\right)^{2 w_{1}} \oplus q\left(T_{t_{H}}^{t}\left(L H\left(\widetilde{a}_{2}\right)\right)\right)^{2 w_{2}}\right) \\
& \otimes\left(p\left(T_{t_{H}}^{t}\left(L H\left(\widetilde{a}_{2}\right)\right)\right)^{2 w_{2}} \oplus q\left(T_{t_{H}}^{t}\left(L H\left(\widetilde{a}_{2}\right)\right)\right)^{2 w_{2}}\right)
\end{aligned}
$$

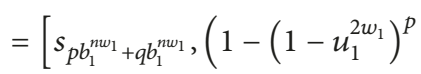

$$
\begin{aligned}
& \left.\left.\cdot\left(1-u_{1}^{2 w_{1}}\right)^{q},\left(1-\left(1-v_{1}\right)^{2 w_{1}}\right)^{p}\left(1-\left(1-v_{1}\right)^{2 w_{1}}\right)^{q}\right)\right] \\
& \otimes\left[s_{p b_{1}^{n w_{1}}+q b_{2}^{n w_{2}}},\left(1-\left(1-u_{1}^{2 w_{1}}\right)^{p}\right.\right. \\
& \left.\left.\cdot\left(1-u_{2}^{n w_{2}}\right)^{q},\left(1-\left(1-v_{1}\right)^{2 w_{1}}\right)^{p}\left(1-\left(1-v_{2}\right)^{2 w_{2}}\right)^{q}\right)\right]
\end{aligned}
$$

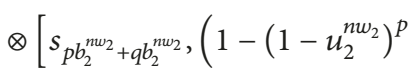

$$
\begin{aligned}
& \left.\left.\cdot\left(1-u_{2}^{n w_{2}}\right)^{q},\left(1-\left(1-v_{2}\right)^{2 w_{2}}\right)^{p}\left(1-\left(1-v_{2}\right)^{2 w_{2}}\right)^{q}\right)\right] \\
& =\left[s _ { i = 1 } ^ { 2 } \prod _ { j = i } ^ { 2 } ( p b _ { i } ^ { 2 w _ { i } } + q b _ { j } ^ { 2 w _ { j } } ) \left(\prod_{i=1}^{2} \prod_{j=i}^{2}\right.\right. \\
& \cdot\left(1-\left(1-u_{i}^{2 w_{i}}\right)^{p}\left(1-u_{j}^{2 w_{j}}\right)^{q}\right), 1-\prod_{i=1}^{2} \prod_{j=i}^{2} \\
& \left.\left.\cdot\left(1-\left(1-\left(1-v_{i}\right)^{2 w_{i}}\right)^{p}\left(1-\left(1-v_{j}\right)^{2 w_{j}}\right)^{q}\right)\right)\right]
\end{aligned}
$$

If Eq. (B.2) for $n=k$, ie.,

$$
\begin{aligned}
\bigotimes_{i=1}^{k} \bigotimes_{j=i}^{k}\left(p\left(T_{t_{H}}^{t}\left(\operatorname{LH}\left(\widetilde{a}_{i}\right)\right)\right)^{k w_{i}} \oplus q\left(T_{t_{H}}^{t}\left(\operatorname{LH}\left(\widetilde{a}_{j}\right)\right)\right)^{k w_{j}}\right) \\
\quad=\left[s \prod_{i=1}^{k} \Pi_{j=i}^{k}\left(p b_{i}^{k w_{i}}+q b_{j}^{k w_{j}}\right)^{,}\right. \\
\quad\left(\prod_{i=1}^{k} \prod_{j=i}^{k}\left(1-\left(1-u_{i}^{k w_{i}}\right)^{p}\left(1-u_{j}^{k w_{j}}\right)^{q}\right), 1\right. \\
\left.\left.-\prod_{i=1}^{k} \prod_{j=i}^{k}\left(1-\left(1-\left(1-v_{i}\right)^{k w_{i}}\right)^{p}\left(1-\left(1-v_{j}\right)^{k w_{j}}\right)^{q}\right)\right)\right]
\end{aligned}
$$


Then, for $\mathrm{n}=\mathrm{k}+1$, we obtain

$$
\begin{aligned}
& \bigotimes_{i=1}^{k+1} \bigotimes_{j=i}^{k+1}\left(p\left(T_{t_{H}}^{t}\left(L H\left(\tilde{a}_{i}\right)\right)\right)^{(k+1) w_{i}}\right. \\
& \left.\oplus q\left(T_{t_{H}}^{t}\left(L H\left(\tilde{a}_{j}\right)\right)\right)^{(k+1) w_{j}}\right) \\
& =\bigotimes_{i=1}^{k} \bigotimes_{j=i}^{k}\left(p\left(T_{t_{H}}^{t}\left(L H\left(\tilde{a}_{i}\right)\right)\right)^{(k+1) w_{i}}\right.
\end{aligned}
$$

$$
\begin{aligned}
& \left.\oplus q\left(T_{t_{H}}^{t}\left(L H\left(\tilde{a}_{j}\right)\right)\right)^{(k+1) w_{j}}\right) \\
& \otimes\left(\bigotimes _ { i = 1 } ^ { k + 1 } \left(p\left(T_{t_{H}}^{t}\left(L H\left(\tilde{a}_{i}\right)\right)\right)^{(k+1) w_{i}}\right.\right. \\
& \left.\left.\oplus q\left(T_{t_{H}}^{t}\left(L H\left(\tilde{a}_{j}\right)\right)\right)^{(k+1) w_{k+1}}\right)\right)
\end{aligned}
$$

By Eq. (55), (B.4), we can transform (B.5) as

$$
\begin{aligned}
& \bigotimes_{i=1}^{k+1} \bigotimes_{j=i}^{k+1}\left(p\left(T_{t_{H}}^{t}\left(L H\left(\tilde{a}_{i}\right)\right)\right)^{(k+1) w_{i}} \oplus q\left(T_{t_{H}}^{t}\left(L H\left(\tilde{a}_{j}\right)\right)\right)^{(k+1) w_{j}}\right)=\left[s_{\prod_{i=1}^{k} \prod_{j=i}^{k}\left(p b_{i}^{(k+1) w_{i}}+q b_{j}^{(k+1) w_{j}}\right)^{\prime}}\right. \\
& \left.\left(\prod_{i=1}^{k} \prod_{j=i}^{k}\left(1-\left(1-u_{i}^{(k+1) w_{i}}\right)^{p}\left(1-u_{j}^{(k+1) w_{j}}\right)^{q}\right), 1-\prod_{i=1}^{k} \prod_{j=i}^{k}\left(1-\left(1-\left(1-v_{i}\right)^{(k+1) w_{i}}\right)^{p}\left(1-\left(1-v_{j}\right)^{(k+1) w_{j}}\right)^{q}\right)\right)\right] \\
& \otimes\left[\prod_{i=1}^{k+1}\left(p b_{i}^{(k+1) w_{i}}+q b_{j}^{(k+1) w_{k+1}}\right)^{\prime}\right. \\
& \left.\left(\prod_{i=1}^{k+1}\left(1-\left(1-u_{i}^{(k+1) w_{i}}\right)^{p}\left(1-u_{j}^{(k+1) w_{k+1}}\right)^{q}\right), 1-\prod_{i=1}^{k+1}\left(1-\left(1-\left(1-v_{i}\right)^{(k+1) w_{i}}\right)^{p}\left(1-\left(1-v_{k+1}\right)^{(k+1) w_{k+1}}\right)^{q}\right)\right)\right] \\
& =\left[\sum_{\prod_{i=1}^{k+1} \prod_{j=i}^{k+1}\left(p b_{i}^{(k+1) w_{i}}+q b_{j}^{(k+1) w_{j}}\right)^{\prime}}\left(\prod_{i=1}^{k} \prod_{j=i}^{k+1}\left(1-\left(1-u_{i}^{(k+1) w_{i}}\right)^{p}\left(1-u_{j}^{(k+1) w_{j}}\right)^{q}\right), 1-\prod_{i=1}^{k+1} \prod_{j=i}^{k+1}\left(1-\left(1-\left(1-v_{i}\right)^{(k+1) w_{i}}\right)^{p}\left(1-\left(1-v_{j}\right)^{(k+1) w_{j}}\right)^{q}\right)\right)\right]
\end{aligned}
$$

\section{Furthermore, we have}

$$
\begin{aligned}
& \left(\bigotimes_{i=1}^{n} \bigotimes_{j=i}^{n}\left(p\left(T_{\mathrm{t}_{H}}^{t}\left(L H\left(\widetilde{a}_{i}\right)\right)\right)^{n w_{i}} \oplus q\left(T_{t_{H}}^{t}\left(L H\left(\widetilde{a}_{j}\right)\right)\right)^{n w_{j}}\right)\right)^{2 / n(n+1)}
\end{aligned}
$$

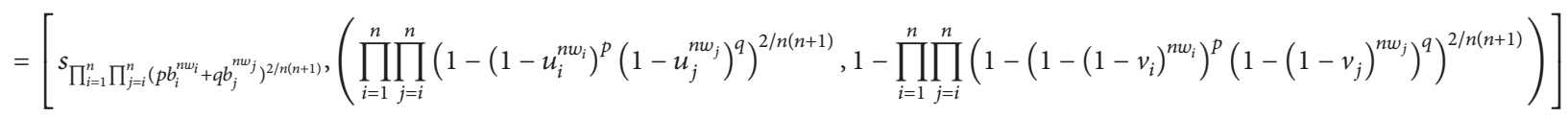

$$
\begin{aligned}
& \frac{1}{p+q}\left(\left(\bigotimes_{i=1}^{n} \bigotimes_{j=i}^{n}\left(p\left(T_{t_{H}}^{t}\left(L H\left(\tilde{a}_{i}\right)\right)\right)^{n w_{i}} \oplus q\left(T_{t_{H}}^{t}\left(L H\left(\tilde{a}_{j}\right)\right)\right)^{n w_{j}}\right)\right)^{2 / n(n+1)}\right) \\
& =\left[\left(1-\left(1-\prod_{i=1}^{n} \prod_{j=i}^{n}\left(1-\left(1-u_{i}^{n w_{i}}\right)^{p}\left(1-u_{j}^{n w_{j}}\right)^{q}\right)^{2 / n(n+1)}\right)^{s_{(1 /(p+q))} \prod_{i=1}^{n} \prod_{j=i}^{n}\left(p+q b_{i}^{n w_{i}}+q b_{j}^{n w_{j}}\right)^{2 / n(n+1)},},\left(1-\prod_{i=1}^{n} \prod_{j=i}^{n}\left(1-\left(1-\left(1-v_{i}\right)^{n w_{i}}\right)^{p}\left(1-\left(1-v_{j}\right)^{n w_{j}}\right)^{q}\right)^{2 / n(n+1)}\right)^{1 /(p+q)}\right)\right]
\end{aligned}
$$


Thus,

$$
\begin{aligned}
& L H^{-1}\left(\frac{1}{p+q}\left(\left(\bigotimes_{i=1}^{n} \bigotimes_{j=i}^{n}\left(p\left(T_{t_{H}}^{t}\left(L H\left(\widetilde{a}_{i}\right)\right)\right)^{n w_{i}} \oplus q\left(T_{t_{H}}^{t}\left(L H\left(\widetilde{a}_{j}\right)\right)\right)^{n w_{j}}\right)\right)^{2 / n(n+1)}\right)\right) \\
& =\left(\left(1-\left(1-\prod_{i=1}^{n} \prod_{j=i}^{n}\left(1-\left(1-u_{i}^{n w_{i}}\right)^{p}\left(1-u_{j}^{n w_{j}}\right)^{q}\right)^{2 / n(n+1)}\right)^{1 /(p+q)},\left(1-\prod_{i=1}^{n} \prod_{j=i}^{n}\left(1-\left(1-\left(1-s_{i}\right)^{n w_{i}}\right)^{p}\left(1-\left(1-v_{j}\right)^{n w_{j}}\right)^{q}\right)^{2 / n(n+1)}\right)^{1 /(p+q)}\right)\right)
\end{aligned}
$$

where

$$
\begin{aligned}
\beta= & \frac{1}{p+q} \prod_{i=1}^{n} \prod_{j=i}^{n}\left(p\left(\Delta^{-1}\left(T_{t_{H}}^{t}\left(L H\left(a_{i}\right)\right)\right)\right)^{n w_{i}}\right. \\
& \left.+q\left(\Delta^{-1}\left(T_{t_{H}}^{t}\left(L H\left(a_{j}\right)\right)\right)\right)^{n w_{j}}\right)^{2 / n(n+1)} .
\end{aligned}
$$

In the following, we prove that the sum of the membership degree and the non-membership degree belongs to $[0,1]$.

Since

$$
\begin{aligned}
& 0 \leq 1-\left(1-\prod_{i=1}^{n} \prod_{j=i}^{n}\left(1-\left(1-u_{i}^{n w_{i}}\right)^{p}\left(1-u_{j}^{n w_{j}}\right)^{q}\right)^{2 / n(n+1)}\right)^{1 /(p+q)} \leq 1, \\
& 0 \leq\left(1-\prod_{i=1}^{n} \prod_{j=i}^{n}\left(1-\left(1-\left(1-v_{i}\right)^{n w_{i}}\right)^{p}\left(1-\left(1-v_{j}\right)^{n w_{j}}\right)^{q}\right)^{2 / n(n+1)}\right)^{1 /(p+q)} \leq 1, \quad u_{i} \leq 1-v_{i} \forall i=1,2, \cdots, n,
\end{aligned}
$$

\section{Thus}

$$
\begin{aligned}
1 & -\left(1-\prod_{i=1}^{n} \prod_{j=i}^{n}\left(1-\left(1-u_{i}^{n w_{i}}\right)^{p}\left(1-u_{j}^{n w_{j}}\right)^{q}\right)^{2 / n(n+1)}\right)^{1 /(p+q)} \\
& +\left(1-\prod_{i=1}^{n} \prod_{j=i}^{n}\left(1-\left(1-\left(1-v_{i}\right)^{n w_{i}}\right)^{p}\left(1-\left(1-v_{j}\right)^{n w_{j}}\right)^{q}\right)^{2 / n(n+1)}\right)^{1 /(p+q)} \leq 1 \\
& -\left(1-\prod_{i=1}^{n} \prod_{j=i}^{n}\left(1-\left(1-\left(1-v_{i}\right)^{n w_{i}}\right)^{p}\left(1-\left(1-v_{j}\right)^{n w_{j}}\right)^{q}\right)^{2 / n(n+1)}\right)^{1 /(p+q)} \\
& +\left(1-\prod_{i=1}^{n} \prod_{j=i}^{n}\left(1-\left(1-\left(1-v_{i}\right)^{n w_{i}}\right)^{p}\left(1-\left(1-v_{j}\right)^{n w_{j}}\right)^{q}\right)^{2 / n(n+1)}\right)^{1 /(p+q)}=1 .
\end{aligned}
$$

This completes the proof of Theorem 27.

$$
\begin{aligned}
& \operatorname{IULDWGGHM} M_{w}^{p, q}\left(\widetilde{a}_{1}, \cdots, \widetilde{a}_{n}\right) \\
& =L H^{-1}\left(\frac{1}{p+q}\left(\left(\bigotimes_{i=1}^{n} \bigotimes_{j=i}^{n}\left(p\left(T_{t_{H}}^{t}\left(L H\left(\widetilde{a}_{i}\right)\right)\right)^{n w_{i}} \oplus q\left(T_{t_{H}}^{t}\left(L H\left(\widetilde{a}_{j}\right)\right)\right)^{n w_{j}}\right)\right)^{2 / n(n+1)}\right)\right)
\end{aligned}
$$

Proof. Since $\widetilde{a}_{i}=\widetilde{a}$ for all $i=1, \cdots, n, w_{1}=w_{2}=\cdots=w_{n}=$ $1 / n$, then we have 


$$
=L H^{-1}\left(\frac{1}{p+q}\left(p\left(T_{t_{H}}^{t}\left(L H\left(\widetilde{a}_{i}\right)\right)\right)^{n \cdot(1 / n)} \oplus q\left(T_{t_{H}}^{t}\left(L H\left(\widetilde{a}_{j}\right)\right)\right)^{n \cdot(1 / n)}\right)\right)=L H^{-1}\left(\left(T_{t_{H}}^{t}(L H(\widetilde{a}))\right)\right)=\widetilde{a} .
$$

\section{The Proof of Theorem 29}

Proof. Since $u_{\alpha_{i}} \leq u_{\beta_{i}}, v_{\alpha_{i}} \geq v_{\beta_{i}}, U L_{\alpha_{i}} \leq U L_{\beta_{i}}$ for all $i$, then we have

$$
\begin{aligned}
1 & -\left(1-\prod_{i=1}^{n} \prod_{j=i}^{n}\left(1-\left(1-u_{\alpha_{i}}^{n w_{i}}\right)^{p}\left(1-u_{\alpha_{j}}^{n w_{j}}\right)^{q}\right)^{2 / n(n+1)}\right)^{1 /(p+q)} \geq 1 \\
& -\left(1-\prod_{i=1}^{n} \prod_{j=i}^{n}\left(1-\left(1-u_{\beta_{i}}^{n w_{i}}\right)^{p}\left(1-u_{\beta_{j}}^{n w_{j}}\right)^{q}\right)^{2 / n(n+1)}\right)^{1 /(p+q)}, \\
& \left(1-\prod_{i=1}^{n} \prod_{j=i}^{n}\left(1-\left(1-\left(1-v_{\alpha_{i}}\right)^{n w_{i}}\right)^{p}\left(1-\left(1-v_{\alpha_{j}}\right)^{n w_{j}}\right)^{q}\right)^{2 / n(n+1)}\right)^{1 /(p+q)} \\
& \geq\left(1-\prod_{i=1}^{n} \prod_{j=i}^{n}\left(1-\left(1-\left(1-v_{\beta_{i}}\right)^{n w_{i}}\right)^{p}\left(1-\left(1-v_{\beta_{j}}\right)^{n w_{j}}\right)^{q}\right)^{2 / n(n+1)}\right)^{1 /(p+q)}, \\
\frac{1}{p+q} & \prod_{i=1}^{n} \prod_{j=i}^{n}\left(p b_{\alpha_{i}}^{n w_{i}}+q b_{\alpha_{j}}^{n w_{j}}\right)^{2 / n(n+1)} \leq \frac{1}{p+q} \prod_{i=1}^{n} \prod_{j=i}^{n}\left(p b_{\beta_{i}}^{n w_{i}}+q b_{\beta_{j}}^{n w_{j}}\right)^{2 / n(n+1)},
\end{aligned}
$$

\section{Thus}

$$
\begin{aligned}
\frac{1}{p+q} & \prod_{i=1}^{n} \prod_{j=i}^{n}\left(p b_{\alpha_{i}}^{n w_{i}}+q b_{\alpha_{j}}^{n w_{j}}\right)^{2 / n(n+1)} \\
& \quad\left(\frac{1}{2}+\frac{1}{2}\left(\begin{array}{c}
1-\left(1-\prod_{i=1}^{n} \prod_{j=i}^{n}\left(1-\left(1-u_{\alpha_{i}}^{n w_{i}}\right)^{p}\left(1-u_{\alpha_{j}}^{n w_{j}}\right)^{q}\right)^{2 / n(n+1)}\right)^{1 /(p+q)} \\
\left.\left.-\left(1-\prod_{i=1}^{n} \prod_{j=i}^{n}\left(1-\left(1-\left(1-v_{\alpha_{i}}\right)^{n w_{i}}\right)^{p}\left(1-\left(1-v_{\alpha_{j}}\right)^{n w_{j}}\right)^{q}\right)^{2 / n(n+1)}\right)^{1 /(p+q)}\right)\right)
\end{array}\right)\right. \\
\leq & \frac{1}{p+q} \prod_{i=1}^{n} \prod_{j=i}^{n}\left(p b_{\beta_{i}}^{n w_{i}}+q b_{\beta_{j}}^{n w_{j}}\right)^{2 / n(n+1)} \\
& \quad\left(\frac{1}{2}+\frac{1}{2}\left(\begin{array}{c}
1-\left(1-\prod_{i=1}^{n} \prod_{j=i}^{n}\left(1-\left(1-u_{\beta_{i}}^{n w_{i}}\right)^{p}\left(1-u_{\beta_{j}}^{n w_{j}}\right)^{q}\right)^{2 / n(n+1)}\right)^{1 /(p+q)} \\
\left.\left.-\left(1-\prod_{i=1}^{n} \prod_{j=i}^{n}\left(1-\left(1-\left(1-v_{\beta_{i}}\right)^{n w_{i}}\right)^{p}\left(1-\left(1-v_{\beta_{j}}\right)^{n w_{j}}\right)^{q}\right)^{2 / n(n+1)}\right)^{1 /(p+q)}\right)\right)
\end{array}\right)\right.
\end{aligned}
$$

i.e. $\operatorname{IULDWGGHM} M_{w}^{p, q}\left(\widetilde{\alpha}_{1}, \cdots, \widetilde{\alpha}_{n}\right) \leq \operatorname{IULDWGGHM} M_{w}^{p, q}\left(\widetilde{\beta}_{1}\right.$, $\left.\cdots, \widetilde{\beta}_{n}\right)$

\section{E. The Proof of Theorem 30}

Proof. Since $\widetilde{\alpha}_{*} \leq \widetilde{\alpha}_{j} \leq \widetilde{\alpha}^{*}$, then we have 


$$
\begin{aligned}
& L H^{-1}\left(\frac{1}{p+q}\left(\left(\bigotimes_{i=1}^{n} \bigotimes_{j=i}^{n}\left(p\left(T_{t_{H}}^{t}\left(L H\left(\widetilde{a}_{*}\right)\right)\right)^{n w_{i}} \oplus q\left(T_{t_{H}}^{t}\left(L H\left(\widetilde{a}_{*}\right)\right)\right)^{n w_{j}}\right)\right)^{2 / n(n+1)}\right)\right) \\
& \quad \leq L H^{-1}\left(\frac{1}{p+q}\left(\left(\bigotimes_{i=1}^{n} \bigotimes_{j=i}^{n}\left(p\left(T_{t_{H}}^{t}\left(L H\left(\widetilde{a}_{i}\right)\right)\right)^{n w_{i}} \oplus q\left(T_{t_{H}}^{t}\left(L H\left(\widetilde{a}_{j}\right)\right)\right)^{n w_{j}}\right)\right)^{2 / n(n+1)}\right)\right) \\
& \quad \leq L H^{-1}\left(\frac{1}{p+q}\left(\left(\bigotimes_{i=1}^{n} \bigotimes_{j=i}^{n}\left(p\left(T_{t_{H}}^{t}\left(L H\left(\widetilde{a}^{*}\right)\right)\right)^{n w_{i}} \oplus q\left(T_{t_{H}}^{t}\left(L H\left(\widetilde{a}^{*}\right)\right)\right)^{n w_{j}}\right)\right)^{2 / n(n+1)}\right)\right)
\end{aligned}
$$

Thus $\widetilde{\alpha}_{*}=\min \left(\widetilde{\alpha}_{1}, \cdots, \widetilde{\alpha}_{n}\right) \leq \operatorname{IULDWGGHM} M_{w}^{p, q}\left(\widetilde{\alpha}_{1}, \cdots\right.$, $\left.\widetilde{\alpha}_{n}\right) \leq \max \left(\widetilde{\alpha}_{1}, \cdots, \widetilde{\alpha}_{n}\right)=\widetilde{\alpha}^{*}$.

\section{Data Availability}

The data used to support the findings of this study are available from the corresponding author upon request.

\section{Conflicts of Interest}

There are no conflicts of interest.

\section{Acknowledgments}

The work was supposed by Natural Science Foundation of China (71771001, 71701001, 61502003 and 71501002). Natural Science Found of Education Department of Anhui province (Nos. KJ2017A026). Anhui Provincial Philosophy and Social Science Planning Youth Foundation (Nos. AHSKQ2016D13). Anhui Provincial Natural Science Foundation (No. 1808085QG211). Statistics and Science Research Foundation of China (No. 2017LZ11).

\section{References}

[1] L. A. Zadeh, "The concept of a linguistic variable and its application to approximate reasoning I," Information Sciences, vol. 8, pp. 199-249, 1975.

[2] L. A. Zadeh, "The concept of a linguistic variable and its application to approximate reasoning-Part II," Information Sciences, vol. 8, pp. 301-357, 1975.

[3] L. A. Zadeh, "The concept of a linguistic variable and its application to approximate reasoning-part III," Information Sciences, vol. 9, no. 1, pp. 43-80, 1975.

[4] F. Herrera and L. Martínez, "A 2-tuple fuzzy linguistic representation model for computing with words," IEEE Transactions on Fuzzy Systems, vol. 8, no. 6, pp. 746-752, 2000.

[5] Z. S. Xu, "A method based on linguistic aggregation operators for group decision making with linguistic preference relations," Information Sciences, vol. 166, no. 1-4, pp. 19-30, 2004.

[6] J. Wang and J. Li, “The multi-criteria group decision making method based on multi-granularity intuitionistic two semantics," Sci Tech Inf, vol. 33, pp. 8-9, 2009.
[7] R. Rodríguez, M. Martínez L, and F. Herrera, "Hesitant fuzzy linguistic term sets, Foundations of Intelligent Systems," Springer Berlin Heidelberg, pp. 287-295, 2011.

[8] P. Ji, H. Zhang, and J. Wang, "A Projection-Based Outranking Method with Multi-Hesitant Fuzzy Linguistic Term Sets for Hotel Location Selection," Cognitive Computation, 2018.

[9] X. Wang, H. Peng, and J. Wang, "Hesitant linguistic intuitionistic fuzzy sets and their application in multicriteria decisionmaking problems," International Journal for Uncertainty Quantification, vol. 8, no. 4, pp. 321-341, 2018.

[10] J. M. Mendel, "An architecture for making judgments using computing with words," International Journal of Applied Mathematics and Computer Science, vol. 12, pp. 325-336, 2002.

[11] F. Herrera, E. Herrera-Viedma, and L. Martínez, "A fuzzy linguistic methodology to deal with unbalanced linguistic term sets," IEEE Transactions on Fuzzy Systems, vol. 16, no. 2, pp. 354370, 2008.

[12] Ł. Bartczuk, P. Dziwiński, and J. T. Starczewski, "A New Method for Dealing with Unbalanced Linguistic Term Set," in Artificial Intelligence and Soft Computing, vol. 7267 of Lecture Notes in Computer Science, pp. 207-212, Springer Berlin Heidelberg, Berlin, Heidelberg, 2012.

[13] Y. Dong, C.-C. Li, and F. Herrera, "Connecting the linguistic hierarchy and the numerical scale for the 2-tuple linguistic model and its use to deal with hesitant unbalanced linguistic information," Information Sciences, vol. 367-368, pp. 259-278, 2016.

[14] Y. Dong, Y. Wu, H. Zhang, and G. Zhang, "Multi-granular unbalanced linguistic distribution assessments with interval symbolic proportions," Knowledge-Based Systems, vol. 82, pp. 139-151, 2015.

[15] B. Wang, J. Liang, Y. Qian, and C. Dang, "A normalized numerical scaling method for the unbalanced multi-granular linguistic sets," International Journal of Uncertainty, Fuzziness and Knowledge-Based Systems, vol. 23, no. 2, pp. 221-243, 2015.

[16] L. Jiang, H. Liu, and J. Cai, "The power average operator for unbalanced linguistic term sets," Information Fusion, vol. 22, pp. 85-94, 2015.

[17] F. Mata, L. G. Perez, F. Chiclana, and E. Herrera-Viedma, "Aggregation of unbalanced fuzzy linguistic information in decision problems based on Type-1 OWA operator," in Proceedings of the IEEE International Conference on Fuzzy Systems, FUZZ-IEEE 2015, Turkey, August 2015. 
[18] Y. Dong, C.-C. Li, and F. Herrera, "An optimization-based approach to adjusting unbalanced linguistic preference relations to obtain a required consistency level," Information Sciences, vol. 292, pp. 27-38, 2015.

[19] Y. Dong, C.-C. Li, Y. Xu, and X. Gu, "Consensus-Based Group Decision Making Under Multi-granular Unbalanced 2-Tuple Linguistic Preference Relations," Group Decision and Negotiation, vol. 24, no. 2, pp. 217-242, 2014.

[20] M. Wang and J. Wang, "New online recommendation approach based on unbalanced linguistic label with integrated cloud," Kybernetes, 2018.

[21] E. Herrera-Viedma and A. G. López-Herrera, "A model of an information retrieval system with unbalanced fuzzy linguistic information," International Journal of Intelligent Systems, vol. 22, no. 11, pp. 1197-1214, 2007.

[22] F. J. Cabrerizo, I. J. Pérez, and E. Herrera-Viedma, "Managing the consensus in group decision making in an unbalanced fuzzy linguistic context with incomplete information," KnowledgeBased Systems, vol. 23, no. 2, pp. 169-181, 2010.

[23] D. Meng and Z. Pei, "On weighted unbalanced linguistic aggregation operators in group decision making," Information Sciences, vol. 223, pp. 31-41, 2013.

[24] L. Martínez, M. Espinilla, J. Liu, L. G. Pérez, and P. J. Sánchez, "An evaluation model with unbalanced linguistic information applied to olive oil sensory evaluation," Journal of MultipleValued Logic and Soft Computing, vol. 15, no. 2-3, pp. 229-251, 2009.

[25] H. Peng and J. Wang, "A Multicriteria Group Decision-Making Method Based on the Normal Cloud Model With Zadeh's Znumbers," IEEE Transactions on Fuzzy Systems, 2018.

[26] X. Zhang, H. Zhang, and J. Wang, "Discussing incomplete 2-tuple fuzzy linguistic preference relations in multi-granular linguistic MCGDM with unknown weight information," Soft Computing, 2017.

[27] Q. Wu, P. Wu, L. Zhou G, H. Chen Y, and X. Guan J, "Some new Hamacher aggregation operators under single-valued neutrosophic 2-tuple linguistic environment and their applications to multi-attribute group decision making," Computers \& Industrial Engineering, pp. 116-144, 2018.

[28] Z. Xu, "Dependent OWA operators," Lecture Notes in Computer Science (including subseries Lecture Notes in Artificial Intelligence and Lecture Notes in Bioinformatics): Preface, vol. 3885, pp. 172$178,2006$.

[29] Z. Xu, "Dependent uncertain ordered weighted aggregation operators," Information Fusion, vol. 9, no. 2, pp. 310-316, 2008.

[30] G. W. Wei, "Method of uncertain linguistic multiple group decision making based on dependent aggregation operators," Journal of Systems Engineering and Electronics, vol. 32, pp. 764769, 2010.

[31] G. Wei and X. Zhao, "Some dependent aggregation operators with 2-tuple linguistic information and their application to multiple attribute group decision making," Expert Systems with Applications, vol. 39, no. 5, pp. 5881-5886, 2012.

[32] S. Sykora, Generalized Heronian Means II, Sykora S. Stans Library, 2009.

[33] P. D. Liu, Z. M. Liu, and X. Zhang, "Some intuitionistic uncertain linguistic Heronian mean operators and their application to group decision making," Applied Mathematics and Computation, vol. 230, pp. 570-586, 2014.

[34] S.-M. Yu, H. Zhou, X.-H. Chen, and J.-Q. Wang, "A multicriteria decision-making method based on Heronian mean operators under a linguistic hesitant fuzzy environment," AsiaPacific Journal of Operational Research, vol. 32, no. 5, article 1550035, Article ID 1550035, 2015.

[35] D. Isern, L. Marin, A. Valls, and A. Moreno, “The unbalanced linguistic ordered weighted averaging operator," in Proceedings of the IEEE International Conference on Fuzzy Systems (FUZZ '10), pp. 1-8, Barcelona, Spain, July 2010.

[36] B. Han, H. Chen, J. Zhu, and J. Liu, "An Approach to Linguistic Multiple Attribute Decision-Making Based on Unbalanced Linguistic Generalized Heronian Mean Aggregation Operator," Computational Intelligence and Neuroscience, vol. 2018, Article ID 1404067, 25 pages, 2018.

[37] B. Han, H. Chen, L. Zhou G, J. Liu P, Z. Tao F, and J. Zhu $\mathrm{M}$, "eneralized dependent unbalanced linguistic aggregation operators and their applications in multiple-attribute decision making," Control and Decision, vol. 23, 836, no. 5, p. 844, 2017.

[38] P. Liu, "Some generalized dependent aggregation operators with intuitionistic linguistic numbers and their application to group decision making," Journal of Computer and System Sciences, vol. 79, no. 1, pp. 131-143, 2013.

[39] O. Cordón, F. Herrera, and I. Zwir, "Linguistic modeling by hierarchical systems of linguistic rules," IEEE Transactions on Fuzzy Systems, vol. 10, no. 1, pp. 2-20, 2002.

[40] L. Martínez, R. M. Rodriguez, and F. Herrera, A 2-tuple linguistic model computing with words in decision making, Springer International Publishing Switzerland, 2015.

[41] F. Herrera and L. Martínez, "A model based on linguistic 2tuples for dealing with multigranular hierarchical linguistic contexts in multi-expert decision-making," IEEE Transactions on Systems, Man, and Cybernetics, Part B: Cybernetics, vol. 31, no. 2, pp. 227-234, 2001. 


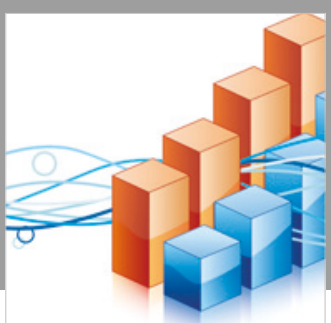

Advances in

Operations Research

\section{-n-m}
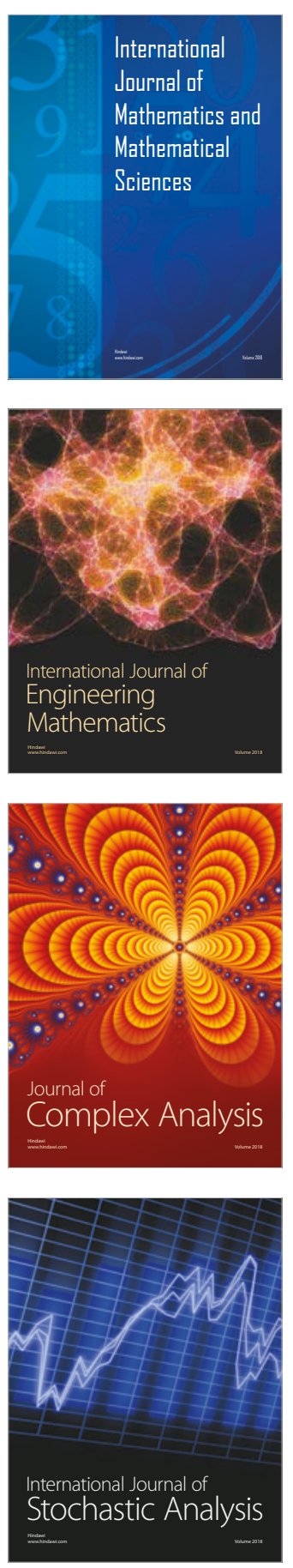
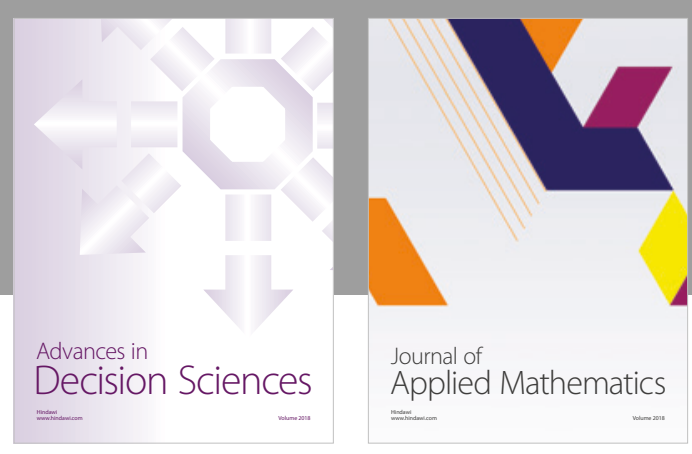

Journal of

Applied Mathematics
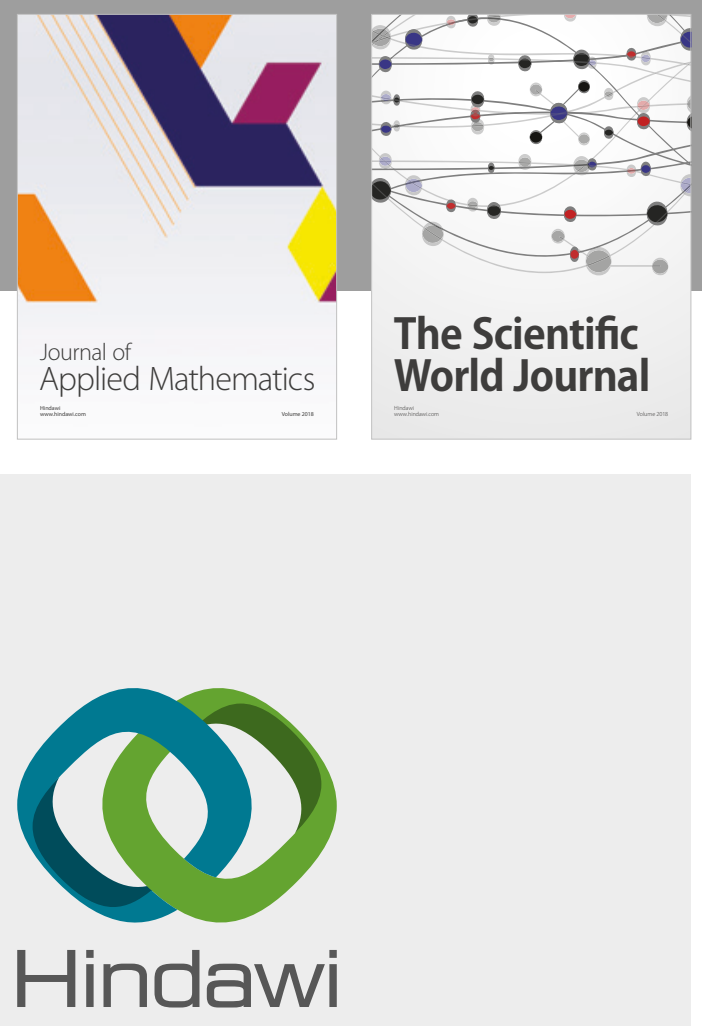

Submit your manuscripts at

www.hindawi.com

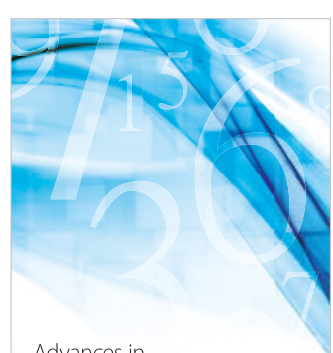

Advances in
Numerical Analysis
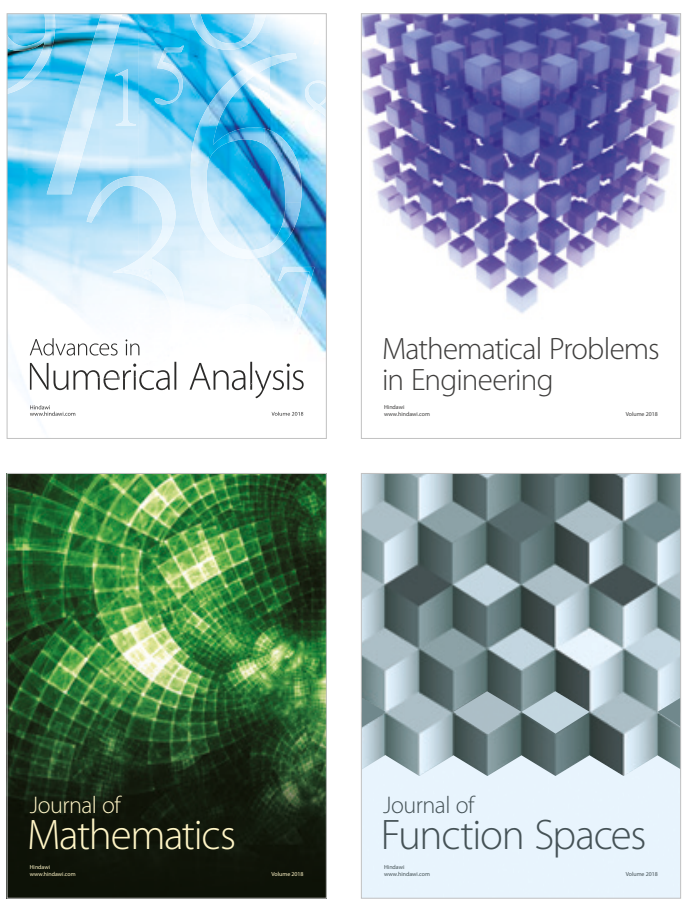

Mathematical Problems in Engineering

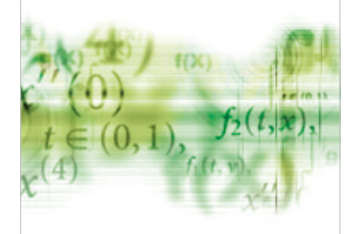

International Journal of

Differential Equations

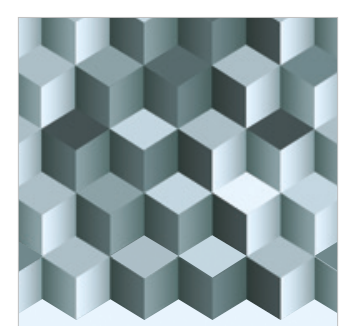

Journal of

Function Spaces

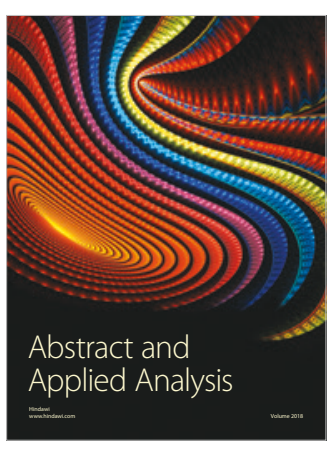

The Scientific

World Journal

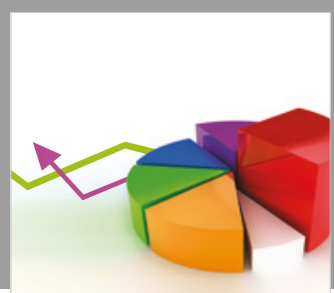

Journal of

Probability and Statistics
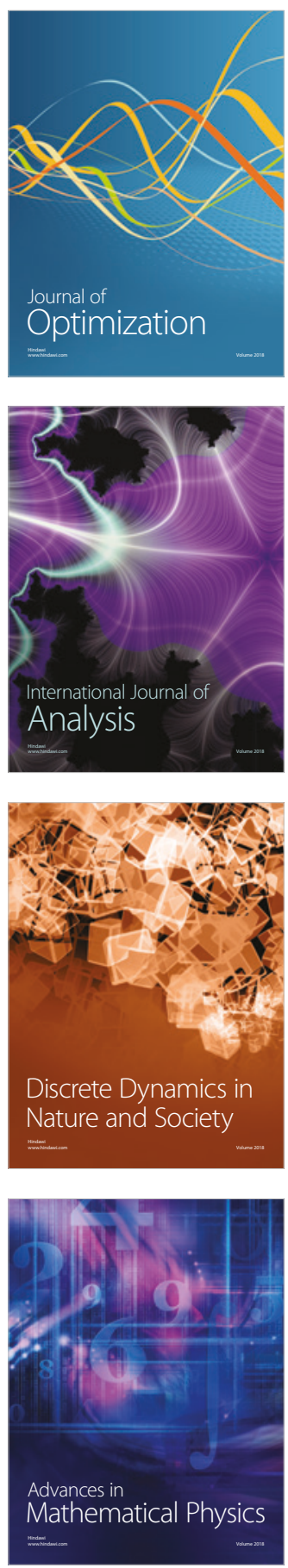